\title{
High-Order Coupled Cluster Method (CCM) Calculations for Quantum Magnets with Valence-Bond Ground States
}

\author{
D.J.J. Farnell $•$ J. Richter $\cdot$ R. Zinke $\cdot$ R.F. Bishop
}

Received: 28 July 2008 / Accepted: 25 February 2009 / Published online: 14 March 2009

(C) Springer Science+Business Media, LLC 2009

\begin{abstract}
In this article, we prove that exact representations of dimer and plaquette valencebond ket ground states for quantum Heisenberg antiferromagnets may be formed via the usual coupled cluster method (CCM) from independent-spin product (e.g. Néel) model states. We show that we are able to provide good results for both the ground-state energy and the sublattice magnetization for dimer and plaquette valence-bond phases within the CCM. As a first example, we investigate the spin-half $J_{1}-J_{2}$ model for the linear chain, and we show that we are able to reproduce exactly the dimerized ground (ket) state at $J_{2} / J_{1}=0.5$. The dimerized phase is stable over a range of values for $J_{2} / J_{1}$ around 0.5 , and results for the ground-state energies are in good agreement with the results of exact diagonalizations of finite-length chains in this regime. We present evidence of symmetry breaking by considering the ket- and bra-state correlation coefficients as a function of $J_{2} / J_{1}$. A radical change is also observed in the behavior of the CCM sublattice magnetization as we enter the dimerized phase. We then consider the Shastry-Sutherland model and demonstrate that the CCM can span the correct ground states in both the Néel and the dimerized phases. Once again, very good results for the ground-state energies are obtained. We find CCM critical points of the bra-state equations that are in agreement with the known phase transition point for
\end{abstract}

D.J.J. Farnell ( $\varangle)$

Academic Department of Radiation Oncology, Division of Cancer Studies, Faculty of Medical and Human Science, University of Manchester, c/o Christie Hospital NHS Foundation Trust, Manchester M20 4BX, UK

e-mail: damian.farnell@manchester.ac.uk

J. Richter · R. Zinke

Institut für Theoretische Physik, Otto-von-Guericke Universität Magdeburg, P.O.B. 4120,

39016 Magdeburg, Germany

R.F. Bishop

School of Physics and Astronomy, Schuster Building, The University of Manchester,

Manchester M13 9PL, UK

R.F. Bishop

School of Physics and Astronomy, University of Minnesota, 116 Church Street S.E., Minneapolis, MN 55455, USA 
this model. The results for the sublattice magnetization remain near to the "true" value of zero over much of the dimerized regime, although they diverge exactly at the critical point. Finally, we consider a spin-half system with nearest-neighbor bonds for an underlying lattice corresponding to the magnetic material $\mathrm{CaV}_{4} \mathrm{O}_{9}(\mathrm{CAVO})$. We show that we are able to provide excellent results for the ground-state energy in each of the plaquette-ordered, Néelordered, and dimerized regimes of this model. The exact plaquette and dimer ground states are reproduced by the CCM ket state in their relevant limits. Furthermore, we estimate the range over which the Néel order is stable, and we find the CCM result is in reasonable agreement with the results obtained by other methods. Our new approach has the dual advantages that it is simple to implement and that existing CCM codes for independent-spin product model states may be used from the outset. Furthermore, it also greatly extends the range of applicability to which the CCM may be applied. We believe that the CCM now provides an excellent choice of method for the study of systems with valence-bond quantum ground states.

Keywords Quantum magnetism · Coupled cluster method (CCM) · Valence-Bond crystals

\section{Introduction}

Lattice quantum spin models not only provide useful models of many physically realizable magnetic systems but also serve as prototypical models of strongly interacting quantum many-body systems. Indeed, the basic models of quantum magnets are given by lattice spin models that often display rich quantum phase transitions between ground states of different order as some control parameter is varied. Their collective behavior is extremely complex due to the presence of strong quantum effects. Furthermore, the underlying crystallographic lattices for these materials may exhibit complex symmetries. Their rich phase diagrams include exotic phases of novel quantum order due to the strong interplay between competing interactions and large quantum fluctuations. For all of these reasons they have naturally provided an excellent test-bed where the various methods of quantum many-body theory can be applied and further refined.

Of particular interest is the formation of dimer- and plaquette-ordered singlet ground states (so-called valence-bond crystal (VBC) states) in quantum spin systems. Often, the formation of enhanced dimer or plaquette correlations is driven by frustration, which can increase quantum fluctuations and which may result in such gapped rotationally-invariant quantum paramagnetic states [1-27]. Usually, VBC states are complicated quantum manybody states, see, e.g., the Heisenberg antiferromagnet on the star lattice [12, 26, 27]. However, for certain systems the VBC states are simple exact product eigenstates of the underlying Heisenberg interaction Hamiltonian. Examples for the appearance of such exact VBC product eigenstates are the spin-half $J_{1}-J_{2}$ model on the linear chain [1-10] at the point $J_{2} / J_{1}=0.5$ (the so-called Majumdar-Ghosh point) and the Shastry-Sutherland model [11-25]. Furthermore, it is often useful to distinguish between VBC phases that have the same translational symmetry as the spin Hamiltonian and those that spontaneously break the symmetry of the underlying spin lattice. Examples of the former case are the ShastrySutherland model and the Heisenberg antiferromagnet on the star lattice, whereas the $J_{1}-J_{2}$ model on the linear chain is an example of spontaneous symmetry breaking.

Another mechanism for the formation of non-magnetic dimer or plaquette VBC ground states that does not involve frustration is the competition between non-equivalent antiferromagnetic nearest-neighbor bonds. This may lead to the formation of local singlets of 
two (or four) coupled spins if the strengths of the non-equivalent bonds differ sufficiently [12, 28-39]. By contrast to frustration, which yields competition in quantum as well as in classical spin systems, this type of competition is present only in quantum systems. The symmetry of the ground state follows the symmetry of the Hamiltonian in such cases. An example of this is given by the nearest-neighbor models for the magnetic material $\mathrm{CaV}_{4} \mathrm{O}_{9}$ of Refs. [28-34] in which one finds two non-equivalent antiferromagnetic nearestneighbor bonds $J$ and $J^{\prime}$ belonging to dimers $\left(J^{\prime}\right)$ or to four-spin plaquettes $(J)$. If these non-equivalent nearest-neighbor bonds are different in strength then local singlet formation may destroy Néel long-range order. As $J^{\prime} / J$ becomes large, local singlets are formed on dimers. Alternatively, local four-spin singlets on the plaquettes are formed for strong plaquette bonds $J$. Another example studied in the literature is the so-called $J-J^{\prime}$ model on the square lattice, i.e., a model with a regular distribution of two different nearest-neighbor bonds on the square lattice [35-40]. In both cases, the formation of local singlets suppresses the magnetic Néel long-range order. However, the VBC ground state is now a complex many-body state; with a simple product VBC state appearing only in the limits $J^{\prime} / J \rightarrow \infty$ and $J / J^{\prime} \rightarrow \infty$.

In this article we focus on the application of the coupled cluster method (CCM) to quantum Heisenberg antiferromagnets having VBC ground-state phases. The CCM $[25,37,39,41-92]$ is one of the most powerful and most widely applied techniques of modern-day microscopic quantum many-body theory. The CCM allows the inclusion of multi-particle correlations into the ground- and excited-state wave functions in a controlled and systematic manner. It has been applied to a great variety of different lattice quantum spin systems with great success. In particular, it has been used successfully with model (or reference) states built by independent-spin product states for which the choice of state for the spin on each site is formally independent of the choice of all others. Often for these independent-spin product model states the use of collinear states, such as the Néel state, is possible where all spins are aligned parallel or antiparallel to one axis (e.g., the $z$-axis), see, Refs. [55-61, 64-75, 77, 79, 82, 84-92]. However, noncollinear (e.g. spiral) model states can be favorable for certain frustrated spin systems [25, 37, 63, 76, 78, 80, 83, 86]. Multispin correlations are then included systematically on top of the independent-spin product model states.

The CCM for independent-spin product model states may be applied to high orders by using a computational implementation described in Refs. [64, 68, 72, 77, 80, 81]. In particular, it may be applied to lattices of complex crystallographic symmetry. Furthermore, it is not constrained to systems with spin quantum number $s=1 / 2$.

In previous work, non-classical VBC ordering has also been considered using the CCM by employing directly valence-bond model states, i.e. two- or four-spin singlet product states [62]. However, this earlier approach involves the direct use of products of localized states (e.g., two-spin dimers or four-spin plaquettes) in the model state. Hence, this approach requires that a whole new matrix-operator formalism be created for each new problem. Also, the Hamiltonian and CCM ket- and bra-state operators must be written in terms of this new matrix algebra. The CCM equations may be derived and solved once the commutation relationships between the operators have been established. Although formally straightforward, this process can be tedious and time-consuming. Furthermore, the existing high-order CCM formalism and codes also need to be amended extensively for each separate model considered.

In this article we use a quite different way to describe VBC states. Starting directly from collinear independent-spin product model states, we discuss how we can form exact local dimer or plaquette ground states within the CCM. This approach has the advantages 
of being conceptually simple and thus also of being easy to implement. Furthermore, we can use directly the existing high-order CCM formalism, computer codes, and extrapolation schemes. After first describing the new methodology, we apply the method to specific spin models considered in the literature that exhibit dimer and plaquette ground states. By comparison with existing results, we demonstrate that the CCM provides good results for the ground-state properties of these systems. We then conclude the article by considering the implications of our results.

\section{Method}

\subsection{CCM Formalism}

The exact ket and bra ground-state energy eigenvectors, $|\Psi\rangle$ and $\langle\tilde{\Psi}|$, of a general manybody system described by a Hamiltonian $H$,

$$
H|\Psi\rangle=E_{g}|\Psi\rangle ; \quad\langle\tilde{\Psi}| H=E_{g}\langle\tilde{\Psi}|,
$$

are parametrized within the normal (NCCM) version of the single-reference CCM as follows:

$$
\begin{aligned}
& |\Psi\rangle=\mathrm{e}^{S}|\Phi\rangle ; \quad S=\sum_{I \neq 0} \mathcal{S}_{I} C_{I}^{+}, \\
& \langle\tilde{\Psi}|=\langle\Phi| \tilde{S} \mathrm{e}^{-S} ; \quad \tilde{S}=1+\sum_{I \neq 0} \tilde{\mathcal{S}}_{I} C_{I}^{-} .
\end{aligned}
$$

The normalized single model or reference state $|\Phi\rangle$ is defined with respect to a suitable set of (mutually commuting) many-spin creation operators $\left\{C_{I}^{+}\right\}$. We note that the model states are generally related to the classical ground states of the lattice spin system. These states are products of single-spin eigenstates of some appropriately defined operator $s_{i}^{z}$ whose direction in a set of global axes can differ from site to site, e.g., a typical such states is: $|\Phi\rangle=\cdots \otimes|\uparrow\rangle \otimes|\downarrow\rangle \otimes|\uparrow\rangle \otimes|\downarrow\rangle \otimes \cdots$ (Néel state). However, we remark also that planar model states or spiral model states in the global spin coordinate axes $(|\Phi\rangle=\cdots \otimes|\rightarrow\rangle \otimes|\leftarrow\rangle \otimes|\rightarrow\rangle \otimes|\leftarrow\rangle \otimes \cdots$ and $|\Phi\rangle=\cdots \otimes|\uparrow\rangle \otimes|\nearrow\rangle \otimes|\rightarrow\rangle \otimes|\searrow\rangle$ $\otimes|\downarrow\rangle \otimes|\swarrow\rangle \otimes \cdots$, respectively) may also be considered. In order to make the CCM calculations easier to carry out in practice, we generally rotate the local axes of the spins so that they all appear notationally to point in the downwards $z$-direction. The model state is then given by: $|\Phi\rangle=\cdots \otimes|\downarrow\rangle \otimes|\downarrow\rangle \otimes|\downarrow\rangle \otimes|\downarrow\rangle \otimes \cdots$. The interested reader is referred to Refs. [25, 37, 63, 76, 78, 80, 83, 86] for more details about spiral model states and Refs. $[65,89]$ for more details about planar model states.

The operators $C_{I}^{+} \equiv\left(C_{I}^{-}\right)^{\dagger}$, with $C_{0}^{+} \equiv 1$, have the property that $\langle\Phi| C_{I}^{+}=0=$ $C_{I}^{-}|\Phi\rangle \forall I \neq 0$. They form a complete set of multi-spin creation operators with respect to the model state $|\Phi\rangle$. Thus, the creation operators are represented simply as a product of spin-raising operators $s_{k}^{+} \equiv s_{k}^{x}+\mathrm{i} s_{k}^{y}$ over the set of lattice sites $\{k\}$ after rotation of the local frames such that all spins appear to point downwards, as described above. The creation operators are now given by $C_{I}^{+} \equiv s_{i_{1}}^{+} s_{i_{2}}^{+} \cdots s_{i_{1}}^{+}$. We note that the definitions of (2) imply the normalization $\langle\tilde{\Psi} \mid \Psi\rangle=\langle\Phi \mid \Phi\rangle=1$.

The determination of the correlation coefficients $\left\{\mathcal{S}_{I}, \tilde{\mathcal{S}}_{I}\right\}$ is achieved by requiring the ground-state energy expectation functional $\bar{H}\left(\left\{\mathcal{S}_{I}, \tilde{\mathcal{S}}_{I}\right\}\right) \equiv\langle\tilde{\Psi}|H| \Psi\rangle=\left\langle\Phi\left|\tilde{S} \mathrm{e}^{-S} H \mathrm{e}^{S}\right| \Phi\right\rangle$ to 
be stationary with respect to variations in each of the (independent) variables of the full set. We thereby derive the following coupled set of equations:

$$
\begin{aligned}
& \delta \bar{H} / \delta \tilde{\mathcal{S}}_{I}=0 \quad \Rightarrow \quad\left\langle\Phi\left|C_{I}^{-} \mathrm{e}^{-S} H \mathrm{e}^{S}\right| \Phi\right\rangle=0, \quad \forall I \neq 0 ; \\
& \delta \bar{H} / \delta \mathcal{S}_{I}=0 \quad \Rightarrow \quad\left\langle\Phi\left|\tilde{S} \mathrm{e}^{-S}\left[H, C_{I}^{+}\right] \mathrm{e}^{S}\right| \Phi\right\rangle=0, \quad \forall I \neq 0 .
\end{aligned}
$$

Equation (3) also shows that the ground-state energy at the stationary point has the simple form

$$
\begin{aligned}
E_{g} & \equiv\langle\tilde{\Psi}|H| \Psi\rangle=\left\langle\Phi\left|\tilde{S} \mathrm{e}^{-S} H \mathrm{e}^{S}\right| \Phi\right\rangle \\
& =\left\langle\Phi\left|\left(1+\sum_{I \neq 0} \tilde{\mathcal{S}}_{I} C_{I}^{-}\right) \mathrm{e}^{-S} H \mathrm{e}^{S}\right| \Phi\right\rangle \\
& =\left\langle\Phi\left|\mathrm{e}^{-S} H \mathrm{e}^{S}\right| \Phi\right\rangle+\sum_{I \neq 0} \tilde{\mathcal{S}}_{I}\left\langle\Phi\left|C_{I}^{-} \mathrm{e}^{-S} H \mathrm{e}^{S}\right| \Phi\right\rangle \\
& \Rightarrow \quad E_{g}=\left\langle\Phi\left|\mathrm{e}^{-S} H \mathrm{e}^{S}\right| \Phi\right\rangle .
\end{aligned}
$$

We see that the expectation value of the ground-state energy of (5) contains terms in $S$ only and so it also contains ket-state correlation coefficients only. Generally, however, we need to use both the bra and ket states to find a ground-state expectation value. Indeed, the ground-state energy is the only special case that requires just the ket-state alone. We note also that this (bi-)variational formulation does not lead to an upper bound for $E_{g}$ when the summations for $S$ and $\tilde{S}$ in (2) are truncated, due to the lack of exact hermiticity when such approximations are made. However, it is also important to realize that the HellmannFeynman theorem is preserved in all such approximations [54].

We note that any practical calculation requires an approximation to be made for both $S$ and $\tilde{S}$. The three most common schemes are: (1) the SUB $n$ scheme, in which all correlations involving only $n$ or fewer spins are retained; (2) the SUB $n-m$ sub-approximation, in which all $n$-spin-flip clusters spanning a range of no more than $m$ adjacent lattice sites are retained; and (3) the "localized" LSUB $m$ scheme, in which all multi-spin correlations over distinct locales on the lattice defined by $m$ or fewer contiguous sites are retained.

We use here an order parameter $M \equiv-\left\langle\tilde{\Psi}\left|s^{z}\right| \Psi\right\rangle$ that is thus defined to be the negative of the ground-state expectation value of the operator $s^{z} \equiv \frac{1}{N} \sum_{i=1}^{N} s_{i}^{z}$. Here $N \rightarrow \infty$ is the total number of spins on the lattice and, very importantly, each operator $s_{i}^{z}$ is defined with respect to the local rotated spin axes on lattice site $i$, which are themselves defined by the choice of particular model state $|\Phi\rangle$, as explained previously. Clearly, by definition, for the original model state, the order parameter $M$ is simply $-\left\langle\Phi\left|s^{z}\right| \Phi\right\rangle=\frac{1}{2}$. Quantum correlations (or fluctuations) in the exact ground state will thus have the effect of decreasing $M$ from this maximal value. For the sake of ease of use and clarity we shall henceforth refer to the order parameter $M$ as the sublattice magnetization, although this terminology is strictly only appropriate for antiferromagnetic ordering on a bipartite lattice. Hence, the sublattice magnetization $M$ in the local axes of the spins in which all spins in the model state $|\Phi\rangle$ point in the negative $z$-direction is given by

$$
M=-\frac{1}{N} \sum_{i=1}^{N}\left\langle\tilde{\Psi}\left|s_{i}^{z}\right| \Psi\right\rangle .
$$

This quantity is easily determined once the bra- and ket-state equations have been solved. 


\subsection{Construction of CCM Valence-Bond Ket Ground States}

Following on from our discussion above of the use of independent-spin product states as CCM model states, we now present a method for creating VBC states within the CCM from such independent-spin product model states. As an example, we consider the Heisenberg model that has a Hamiltonian defined by

$$
H=\sum_{\langle i, j\rangle} \mathbf{s}_{i} \cdot \mathbf{s}_{j},
$$

where the indices $i$ and $j$ run over all lattice sites on a given lattice. The brackets around $\langle i, j\rangle$ indicate that all nearest-neighbor pairs are counted once and once only. For the bipartite lattices considered here (namely, the linear chain, the square lattice, and the "CAVO" lattice), we choose a model state in which nearest-neighbor spins are antiparallel along, say, the $z$-direction. The local frames of the "up" spins are rotated by $180^{\circ}$ so that they point downwards in these local axes. This is achieved by carrying out the following transformation of the local axes of these spins: $s^{x} \rightarrow-s^{x} ; s^{y} \rightarrow s^{y}$; and, $s^{z} \rightarrow-s^{z}$. The model state is now formed from a product purely of "down" spins in the rotated spin coordinates, as described above.

The Heisenberg Hamiltonian is now written in terms of the new spin axes by,

$$
H=-\sum_{\langle i, j\rangle}\left(s_{i}^{z} s_{j}^{z}+\frac{1}{2}\left\{s_{i}^{-} s_{j}^{-}+s_{i}^{+} s_{j}^{+}\right\}\right) .
$$

We note that the total spin in the "global" $z$-direction, $s_{T}^{z}=\sum_{i}^{N} s_{i}^{z}$, is a conserved quantity of the ground state in all of the models studied here.

We now present the method for creating VBC states from independent-spin product model states. Let us consider for a moment the one-dimensional spin-half $J_{1}-J_{2}$ Heisenberg antiferromagnet. The relevant model state is the collinear Néel state [8, 63, 86, 87]. However, as mentioned above, the ground state for $J_{2} / J_{1}$ near to 0.5 breaks the translational lattice symmetry and is two-fold degenerate (see also Sect. 3.1). In order to take into account this property, we have to double the unit cell, i.e. the relevant unit cell has two neighboring sites at points $(0,0,0)$ and $(1,0,0)$ and a single Bravais vector of $(2,0,0)^{T}$. Note, however, that such an explicit increase of the unit cells is not necessary for VBC phases that do not break the translational lattice symmetry. The doubling of the unit cell, now enables us to consider two distinct types of two-spin nearest-neighbor ket-state correlation coefficients; in this way allowing to break the translational lattice symmetry. We call the two nearestneighbor ket-state correlation coefficients $\mathcal{S}_{2}^{a}$ and $\mathcal{S}_{2}^{b}$, where, we define $\mathcal{S}_{2}^{a}$ to connect those nearest-neighbor sites between different unit cells and $\mathcal{S}_{2}^{b}$ to connect those nearest-neighbor sites within each unit cell. With respect to the rotated spin coordinates, we may now construct via (2) a simple dimerized product CCM ket state given by either $\mathcal{S}_{2}^{a}=1$ and $\mathcal{S}_{2}^{b}=0$ or $\mathcal{S}_{2}^{a}=0$ and $\mathcal{S}_{2}^{b}=1$ for $S_{2}=\mathcal{S}_{2}^{a} \sum_{i_{a}} s_{i_{a}}^{+} s_{i_{a}+1}^{+}+\mathcal{S}_{2}^{b} \sum_{i_{b}} s_{i_{b}}^{+} s_{i_{b}+1}^{+}$, and where $i_{a}$ runs over all sites with odd-numbered indices and $i_{b}$ runs over all sites with even-numbered indices. It is obvious, that this choice: (i) breaks the lattice symmetry; and, (ii) represents two different degenerate states. The proof that the above choice for $\mathcal{S}_{2}^{a}, \mathcal{S}_{2}^{b}$ leads to dimerized product states is as follows:

$$
\begin{aligned}
|\Psi\rangle & =\mathrm{e}^{S_{2}}|\Phi\rangle \\
& =\mathrm{e}^{\left(\mathcal{S}_{2}^{a} s_{1}^{+} s_{2}^{+}+\mathcal{S}_{2}^{b} s_{2}^{+} s_{3}^{+}+\mathcal{S}_{2}^{a} s_{3}^{+} s_{4}^{+}+\mathcal{S}_{2}^{b} s_{4}^{+} s_{5}^{+}+\cdots\right)}|\Phi\rangle,
\end{aligned}
$$




$$
\begin{aligned}
\mathcal{S}_{2}^{a}=1 ; \mathcal{S}_{2}^{b}=0 \Rightarrow|\Psi\rangle= & \mathrm{e}^{\left(s_{1}^{+} s_{2}^{+}+s_{3}^{+} s_{4}^{+}+s_{5}^{+} s_{6}^{+}+\cdots\right)}|\Phi\rangle \\
= & \left(1+s_{1}^{+} s_{2}^{+}\right)\left(1+s_{3}^{+} s_{4}^{+}\right)\left(1+s_{5}^{+} s_{6}^{+}\right) \cdots|\Phi\rangle \\
= & \left\{\left|\downarrow_{1} \downarrow_{2}\right\rangle+\left|\uparrow_{1} \uparrow_{2}\right\rangle\right\} \otimes\left\{\left|\downarrow_{3} \downarrow_{4}\right\rangle+\left|\uparrow_{3} \uparrow_{4}\right\rangle\right\} \\
& \otimes \cdots,
\end{aligned}
$$

where the notation $\left|\downarrow_{i}\right\rangle$ and $\left|\uparrow_{i}\right\rangle$ indicates a 'down' or 'up' spin, respectively, localized to site $i$. We note that $\left(s_{i}^{+}\right)^{2}|\Phi\rangle=0 \forall i$ is assumed in (9), which holds true for spin-half systems such as those considered here.

Furthermore, we note that if the local axes of spins on one sublattice are "re-rotated" such that 'down' spins become 'up' spins once again, i.e., so as to regain the Heisenberg Hamiltonian of (7) in the global spin coordinate system, then the dimerized product state becomes the usual product of the nearest-neighbor dimer singlets, namely, $\left(\left|\uparrow_{i} \downarrow_{j}\right\rangle-\left|\downarrow_{i} \uparrow_{j}\right\rangle\right)$ at nearest-neighboring sites $i$ and $j$. Note that if such a dimerized product state becomes the true ground state of a given spin problem (e.g., the spin-half linear chain $J_{1}-J_{2}$ model at $J_{2} / J_{1}=0.5$ - see below for more details) then for any level of LSUB $m(m \geq 2)$ approximation either $\mathcal{S}_{2}^{a}=1$ with all other ket-state correlation coefficients (i.e., including those for $m$ spins with $m \geq 2$ ) equal to zero or $\mathcal{S}_{2}^{b}=1$ with all other ket-state correlation coefficients equal to zero, is a valid ket ground-state solution to this problem.

The situation is different for the bra state. Firstly, we note that the dimerized product bra state being equivalent to the corresponding dimerized ket product state of (9) in rotated coordinates ought to be,

$$
\begin{aligned}
\langle\Psi|= & \left\{\left\langle\downarrow_{1} \downarrow_{2}\right|+\left\langle\uparrow_{1} \uparrow_{2}\right|\right\} \otimes\left\{\left\langle\downarrow_{3} \downarrow_{4}\right|+\left\langle\uparrow_{3} \uparrow_{4}\right|\right\} \otimes \cdots \\
= & \left\{\left\langle\downarrow_{1} \downarrow_{2} \downarrow_{3} \downarrow_{4} \downarrow_{5} \downarrow_{6} \cdots\right|+\left\langle\uparrow_{1} \uparrow_{2} \downarrow_{3} \downarrow_{4} \downarrow_{5} \downarrow_{6} \cdots\right|+\left\langle\downarrow_{1} \downarrow_{2} \uparrow_{3} \uparrow_{4} \downarrow_{5} \downarrow_{6} \cdots\right|\right. \\
& \left.+\cdots+\left\langle\downarrow_{1} \downarrow_{2} \uparrow_{3} \uparrow_{4} \uparrow_{5} \uparrow_{6} \uparrow_{7} \uparrow_{8} \cdots\right|+\cdots+\left\langle\uparrow_{1} \uparrow_{2} \uparrow_{3} \uparrow_{4} \uparrow_{5} \uparrow_{6} \uparrow_{7} \uparrow_{8} \cdots\right|\right\} .
\end{aligned}
$$

We notice now that the modes of action of the spin operators (leftwards) on the bra spin states for $s=1 / 2$ are,

$$
\begin{aligned}
& \langle\downarrow| s^{+}=0 ; \quad\langle\downarrow| s^{-}=\langle\uparrow| \\
& \langle\uparrow| s^{-}=0 ; \quad\langle\uparrow| s^{+}=\langle\downarrow| .
\end{aligned}
$$

Thus, the NCCM ground bra state for LSUB $m$ with $m$ a finite number can only ever contain a maximum of $m$ "up" states in the bra state because of the linear nature of the bra-state operator $\tilde{S}$ in (2). Hence, by contrast to the ket state, within the LSUB $m$ approximation we can never construct an equivalent simple dimerized product bra state using the NCCM except in the exact limit where $m \rightarrow \infty$. However, carrying out CCM calculations in the limit $m \rightarrow \infty$ using computational methods is generally not practical. We note that this problem might be alleviated by using the extended coupled cluster method (ECCM). In this method, the bra state is written in terms of an exponential with respect to both the ketstate and bra-state correlation operators. It is this exponential term that allows such dimer solutions, e.g., for the ket state for the NCCM above. However, we do not discuss the ECCM further in this present paper.

We mention that the sublattice magnetization $M$ (see (6)) for the simple dimerized product ket state (i.e., $\mathcal{S}_{2}^{a}=1$ and all other coefficients equal to zero) is written in terms of only one bra-state correlation coefficient, i.e. $M=\frac{1}{2}-2 \tilde{\mathcal{S}}_{2}^{a}$. 


\section{Results}

\subsection{The Spin-Half $J_{1}-J_{2}$ Model on the Linear Chain}

The Hamiltonian for this spin-half model has nearest-neighbor bonds of strength $J_{1}$ and next-nearest-neighbor bonds of strength $J_{2}$. We use a Néel model state in which nearestneighbor spins on the linear chain are antiparallel. We rotate the spin coordinates of the 'up' spins so that notationally they become 'down' spins in these locally defined axes. The relevant Hamiltonian in rotated coordinates is then given by

$$
H=-J_{1} \sum_{\langle i, j\rangle}\left(s_{i}^{z} s_{j}^{z}+\frac{1}{2}\left\{s_{i}^{-} s_{j}^{-}+s_{i}^{+} s_{j}^{+}\right\}\right)+J_{2} \sum_{\langle\langle i, k\rangle\rangle}\left(s_{i}^{z} s_{k}^{z}+\frac{1}{2}\left\{s_{i}^{+} s_{k}^{-}+s_{i}^{-} s_{k}^{+}\right\}\right),
$$

where $\langle i, j\rangle$ runs over all nearest-neighbor sites on the lattice counting each pair once and once only and $\langle\langle i, k\rangle\rangle$ runs over all next-nearest-neighbor sites on the lattice, again counting each pair once and once only. Henceforth we put $J_{1}=1$ and consider $J_{2}>0$.

The ground-state properties of this system have been studied using methods such as exact diagonalizations [3, 10], DMRG [4-8, 63], CCM [60, 62, 86], and field-theoretical approaches [8] (see Refs. [8,9] for a general review). Note that previous CCM studies of the model considering only independent-spin product model states that conserve the lattice symmetry are reported in Refs. [60,87]. At $J_{2} / J_{1}=0$ we have the unfrustrated Heisenberg antiferromagnet, where the exact solution is provided by the Bethe Ansatz. The ground state is gapless and the spin-spin correlation function $\left\langle\mathbf{s}_{i} \cdot \mathbf{s}_{j}\right\rangle$ decays slowly to zero according to a power-law, i.e. no true Néel-like long-range order is observed. In the region $J_{2} / J_{1}>0$ the nearest-neighbor $\left(J_{1}\right)$ and next-nearest-neighbor interactions $\left(J_{2}\right)$ compete, thus leading to frustration. At $J_{2} / J_{1}=0.2411$ (1) the model exhibits a transition to a two-fold degenerate gapped dimerized phase with an exponential decay of the correlation function $\left\langle\mathbf{s}_{i} \cdot \mathbf{s}_{j}\right\rangle$ [3-6, 8, 9]. This state breaks the translational lattice symmetry. At the Majumdar-Ghosh point $J_{2} / J_{1}=0.5$ there are two degenerate simple exact dimer-singlet product ground states corresponding to the dimerized product state of (9) for the Hamiltonian of $(12)[1,2]$. (We recall that rotated spin coordinates are used in (9).)

We now consider how this model can be treated in the dimerized phase by the CCM via, as presented above, the identification of a special dimerized solution of the CCM equations for a Néel model state. Also as discussed above, we use a doubled unit cell including two neighboring sites for a spin-half system on the linear chain at points $(0,0,0)$ and $(1,0,0)$ and a single Bravais vector $(2,0,0)^{T}$ to take into account the symmetry breaking. There are thus two distinct types of two-spin nearest-neighbor ket-state correlation coefficients and again these are denoted as $\mathcal{S}_{2}^{a}$ and $\mathcal{S}_{2}^{b}$. The exact ground state at $J_{2} / J_{1}=0.5$ of (9) is obtained by setting $\mathcal{S}_{2}^{a}=1$ and all other coefficients equal to zero. Starting from $J_{2} / J_{1}=0.5$ we are able to track this exact solution at $J_{2} / J_{1}=0.5$ within a certain $\mathrm{LSUB} m$ approximation for other values of $J_{2} / J_{1}$ and the results for the nearest-neighbor ket-state correlation coefficients in LSUB12 approximation are presented in Fig. 1. Clearly, we see that the exact dimerized product-state solution for the ket ground state is obtained within LSUB12 level of approximation (and, indeed, at all LSUB $m$ approximation with $m \geq 2$ ) at $J_{2} / J_{1}=0.5$, i.e. $\mathcal{S}_{2}^{a}=1$ and all other coefficients equal to zero. Moving away from $J_{2} / J_{1}=0.5$ we still find a CCM ground state that breaks the lattice symmetry. However, this dimerized state deviates from the simple product state, i.e. $\mathcal{S}_{2}^{a} \neq 1$ and other non-zero coefficients $\mathcal{S}_{I}$ occur. Furthermore, the solution (i.e. $\mathcal{S}_{2}^{a}=\mathcal{S}_{2}^{b}$ ) having full translational symmetry is the only solution below a critical point $J_{2} /\left.J_{1}\right|_{c_{1}}(<0.5)$. Henceforth, we shall refer to this solution below $J_{2} /\left.J_{1}\right|_{c_{1}}$ as 


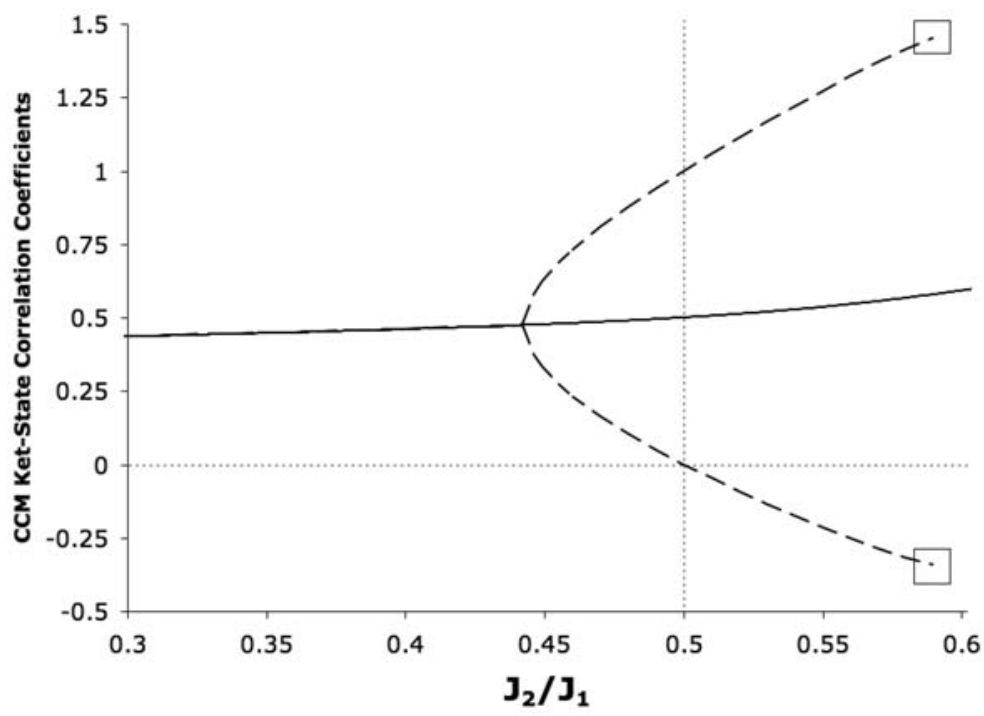

Fig. 1 CCM results at the LSUB12 level of approximation for the ground-state nearest-neighbor ket-state correlation coefficients of the spin-half $J_{1}-J_{2}$ antiferromagnet on the linear chain. The nearest-neighbor coefficients $\mathcal{S}_{2}^{a}$ and $\mathcal{S}_{2}^{b}$ of the symmetry breaking dimerized solution are shown by dashed lines. Results for the usual ('Néel-type') solution (where $\mathcal{S}_{2}^{a}=\mathcal{S}_{2}^{b}$ ) are shown by the full line. Below the (bifurcation) CCM critical point at $J_{2} /\left.J_{1}\right|_{c_{1}}$ there is only the solution with $\mathcal{S}_{2}^{a}=\mathcal{S}_{2}^{b}$. A termination point $J_{2} /\left.J_{1}\right|_{t}$ of the CCM equations for the dimerized solution, at which point the real solution to the CCM equations terminates, is indicated by the boxes

Table 1 CCM results for the positions of the range of the dimerized phase

\begin{tabular}{lll}
\hline LSUB $m$ & $J_{2} /\left.J_{1}\right|_{c_{1}}$ & $J_{2} /\left.J_{1}\right|_{t}$ \\
\hline 2 & 0.4761 & - \\
4 & 0.4745 & 0.5576 \\
6 & 0.4637 & - \\
8 & 0.4568 & 0.7410 \\
10 & 0.4498 & 0.6404 \\
12 & 0.4429 & 0.5956 \\
\hline
\end{tabular}

the "usual ('Néel-type') solution" because previous CCM calculations $[60,62]$ for the $J_{1}-J_{2}$ model have considered the non-symmetry breaking case only. For larger values of $J_{2} / J_{1}$ a CCM termination point is observed at $J_{2} /\left.J_{1}\right|_{t}(>0.5)$, shown by the boxes in Fig. 1 . At this point, the real solution of the CCM dimerized solution is terminated. These CCM results indicate that a dimerized phase exists over a finite range of $J_{2} / J_{1}$, which is in agreement with known results, see e.g. Refs. [4-6, 8, 9]. Qualitatively similar results are observed at other levels of LSUB $m$ approximation for the ket-state correlation coefficients as a function of $J_{2} / J_{1}$. The results for $J_{2} /\left.J_{1}\right|_{c_{1}}$ and $J_{2} /\left.J_{1}\right|_{t}$ are shown in Table 1 . It is obvious that the CCM critical point $J_{2} /\left.J_{1}\right|_{c_{1}}$ becomes smaller (i.e., becomes closer to the true critical point $J_{2} / J_{1}=0.2411(1)[4-6,9]$ ) with higher orders $m$ of LSUB $m$ approximation. However, the critical point $J_{2} /\left.J_{1}\right|_{c_{1}}$ is still significantly too high even at the LSUB12 level of approximation. However, as shown in Ref. [8] the dimerization gap for $J_{2} / J_{1}<0.4$ is very small and it 


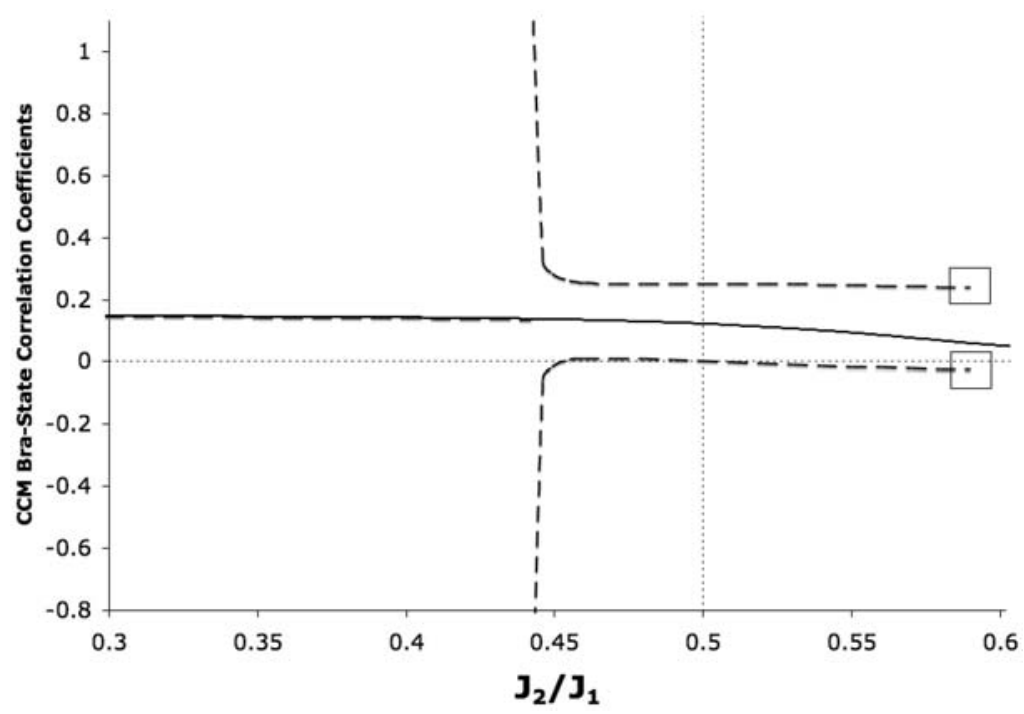

Fig. 2 CCM results at the LSUB12 level of approximation for the ground-state nearest-neighbor bra-state correlation coefficients of the spin-half $J_{1}-J_{2}$ antiferromagnet on the linear chain. The nearest-neighbor coefficients $\tilde{\mathcal{S}}_{2}^{a}$ and $\tilde{\mathcal{S}}_{2}^{b}$ of the dimerized solution are shown by dashed lines. Results for the usual ('Néel-type') solution (where $\tilde{\mathcal{S}}_{2}^{a}=\tilde{\mathcal{S}}_{2}^{b}$ ) are shown by the full line. Below the critical point at $J_{2} /\left.J_{1}\right|_{c_{1}}$ both solutions coincide. Results for these bra-state correlation coefficients diverge at the critical point $J_{2} /\left.J_{1}\right|_{c_{1}}$. A termination point at $J_{2} /\left.J_{1}\right|_{t}$ is shown by the boxes on the right-hand side of the figure

is therefore not very surprising that we do not detect the dimerized phase below $J_{2} / J_{1}<0.4$ using LSUB $m$ approximations with $m \leq 12$. On the side of $J_{2} / J_{1}>0.5$ the existence of termination points can be related to the appearance of incommensurate spiral spin correlations at $J_{2} / J_{1}>0.6[8,10,63,86]$ that are not taken into account in the model state used here.

The nearest-neighbor bra-state correlation coefficient at the LSUB $m$ level of approximation at $J_{2} / J_{1}=0.5$ has $\tilde{\mathcal{S}}_{2}^{a}=1 / 4$ with $m \geq 4$. This is shown in Fig. 2 for the LSUB12 level of approximation. We find that the bra-state solution for the nearest-neighbor correlation coefficients is close to $1 / 4$ over the range $J_{2} /\left.J_{1}\right|_{c_{1}}<J_{2} / J_{1} \leq J_{2} /\left.J_{1}\right|_{t}$. However, we find that the nearest-neighbor correlation coefficient diverges as $J_{2} / J_{1} \rightarrow J_{2} /\left.J_{1}\right|_{c_{1}}$ and this is also shown in Fig. 2. Again, the usual ('Néel-type') solution $\left(\tilde{\mathcal{S}}_{2}^{a}=\tilde{\mathcal{S}}_{2}^{b}\right)$ is obtained for $J_{2} / J_{1}<J_{2} /\left.J_{1}\right|_{c_{1}}$. The upper CCM termination point at $J_{2} /\left.J_{1}\right|_{t}$ is also shown in Fig. 2 by the boxes on the right-hand side of the figure. Once more, qualitatively similar results are observed at other levels of LSUB $m$ approximation for the bra-state correlation coefficients as a function of $J_{2} / J_{1}$.

We now consider the ground-state energy of this system in the dimerized regime. Our results for the new dimer solution and the usual ('Néel-type') solution are shown in Fig. 3. Firstly, we note that the exact ground-state energy of $E_{g} / N=-0.375 J_{1}$ is obtained at the point $J_{2} / J_{1}=0.5$, as expected. We note again that our solution is an exact ground eigenstate at this point. We see that ground-state energy of the usual ('Néel-type') CCM solution in which $\mathcal{S}_{2}^{a}=\mathcal{S}_{2}^{b}$ at the LSUB12 level of approximation actually lies below this exact solution. This indicates (i) that the usual ('Néel-type') CCM solution is a relatively poor choice at this point; and, (ii) that the CCM ground-state energy does not fulfill the variational principle [70]. Furthermore, we see that CCM dimer solution compares extremely well to results 


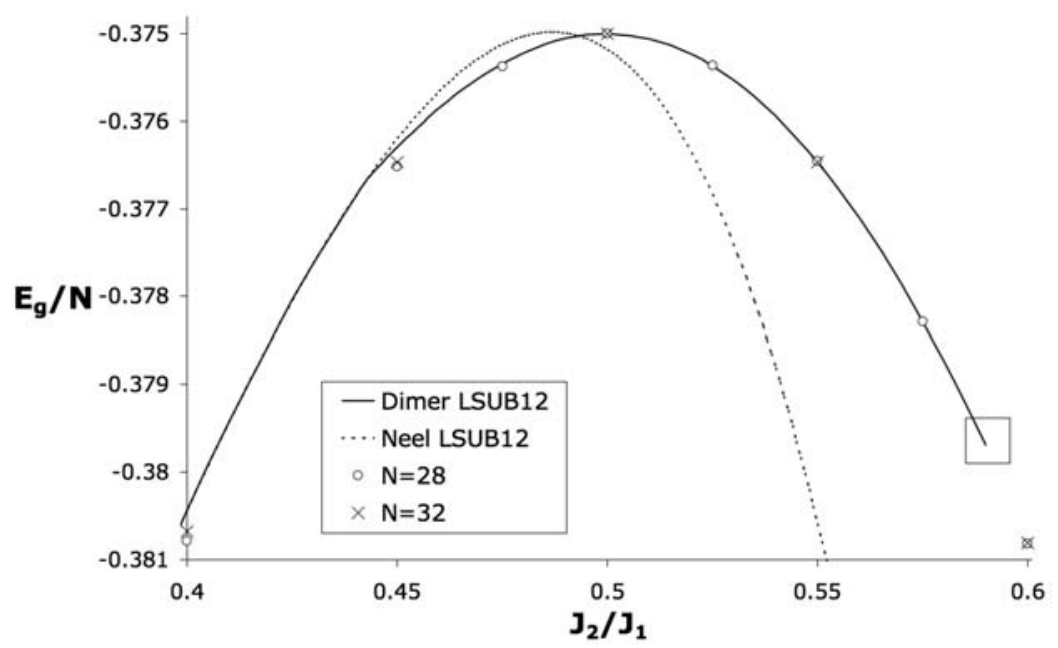

Fig. $3 \mathrm{CCM}$ results for the ground-state energy of the spin-half $J_{1}-J_{2}$ antiferromagnet with $J_{1}=1$ on the linear chain at the LSUB12 level of approximation. The dimerized and usual ('Néel-type') solutions are shown in this figure. Results of exact diagonalizations for $N=28$ and $N=32$ number of sites are also shown. The CCM termination point for the dimerized solution is shown by the box

of exact diagonalizations for $N=28$ and $N=32$ sites in the dimerized regime shown in Fig. 3. It certainly provides far better results than those of the usual ('Néel-type') CCM solution beyond the critical point at $J_{2} /\left.J_{1}\right|_{c_{1}}$.

The results for the sublattice magnetization $M$ of this model are presented graphically in Fig. 4. Since the one-dimensional $J_{1}-J_{2}$ model does not possess Néel long-range order for any value of $J_{1}, J_{2} \geq 0$ the true value is $M=0$. As is known from previous CCM calculations $[60,72,86,87]$, the sublattice magnetization is nonzero (but small) using the usual Néel model state. However, the correct result $M=0$ can be obtained [86, 87] by extrapolating the 'raw' LSUB $m$ data to $m \rightarrow \infty$. Indeed it is obvious that the CCM-LSUB $m$ values for $M$ are non-negligible for Néel model state in the region $J_{2} / J_{1}<J_{2} /\left.J_{1}\right|_{c_{1}}$. It is also obvious that $M$ decreases with the level of approximation $m$ approaching the true value $M=0$, and, that increasing the strength $J_{2}$ of the frustration weakens magnetic order. More interestingly, we find that the sublattice magnetization behaves discontinuously at $J_{2} /\left.J_{1}\right|_{c_{1}}$ by tracking the lattice symmetry-breaking dimerized solution, and then remains near to zero at LSUB10 and LSUB 12 levels of approximation across the entire range $J_{2} /\left.J_{1}\right|_{c_{1}}<J_{2} / J_{1}<J_{2} /\left.J_{1}\right|_{t}$. (At the lower LSUB8 level of approximation the results for the sublattice magnetization differ from zero by a small amount in a small region above $J_{2} / J_{1}>0.5$.) On the other hand, by tracking the usual ('Néel-type') solution $M$ changes continuously with $J_{2}$ and it is larger than for the dimerized solution for $J_{2} /\left.J_{1}\right|_{c_{1}}<J_{2} / J_{1}<0.5$. This behavior of $M$ is another indication that the dimerized CCM solution describes the true physics of the model much better than the usual Néel solution. We note finally that the CCM sublattice magnetization is exactly zero at the Majumdar-Ghosh point $J_{2} / J_{1}=0.5$ at all levels of LSUB $m$ approximation using the dimerized product state.

We remark again that our results for the lower phase transition point $J_{2} /\left.J_{1}\right|_{c_{1}}$ overestimate the position by a factor of two. We re-iterate that we believe that we over-estimate $J_{2} /\left.J_{1}\right|_{c_{1}}$ because the energy gap only becomes large for values of $J_{2} / J_{1}$ of approximately 


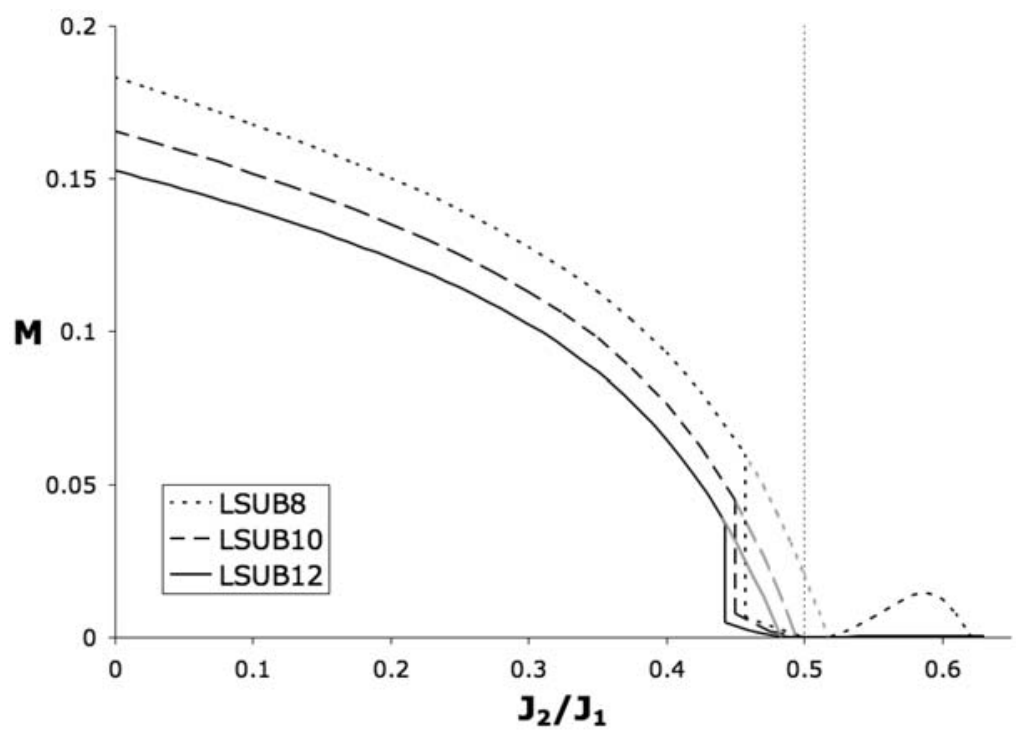

Fig. $4 \mathrm{CCM}$ results for the sublattice magnetization $M$ of the spin-half $J_{1}-J_{2}$ antiferromagnet on the linear chain. Below the critical point at $J_{2} /\left.J_{1}\right|_{c_{1}}$ the results for the usual ('Néel-type') and the dimerized solution coincide. At $J_{2} /\left.J_{1}\right|_{c_{1}}$ the sublattice magnetization of the dimerized solution exhibits a jump, whereas $M$ for the usual ('Néel-type') solution is continuous. Above $J_{2} /\left.J_{1}\right|_{c_{1}}$ the sublattice magnetization of the dimerized solution (black lines) is smaller than that of the usual ('Néel-type') solution (grey lines), except for the LSUB8 in a small region above $J_{2} / J_{1}=0.5$

0.4 [8]. Finally, we note that results given here present the possibility that the CCM might be applied to detect spontaneous symmetry breaking for systems of two or three spatial dimensions, i.e., where other approximate methods become less accurate or may not even be applicable (e.g., such as the DMRG method).

\subsection{The Shastry-Sutherland Antiferromagnet}

Another model that demonstrates dimer order is the Shastry-Sutherland antiferromagnet [11]. The Shastry-Sutherland antiferromagnet is a spin-half Heisenberg model on an underlying square lattice with antiferromagnetic nearest-neighbor bonds $J_{1}$ and with one antiferromagnetic next-nearest-neighbor diagonal bond $J_{2}$ in every second square (plaquette), as shown in Fig. 5. We note that for bonds of equal strength, i.e., $J_{1}=J_{2}$, the Shastry-Sutherland model is equivalent to a Heisenberg model on one of the eleven uniform Archimedean lattices [12]. Interest in this model has been renewed by the discovery of the magnetic material $\mathrm{SrCu}\left(\mathrm{BO}_{3}\right)_{2}[12,13]$ that can be understood in terms of the ShastrySutherland model. The ground state of this model in the limit of small frustration $J_{2} / J_{1} \ll 1$ and large frustration $J_{2} / J_{1} \gg 1$ is well understood. However, the ground-state phase at intermediate values of $J_{2} / J_{1} \approx 1$ is still a matter of discussion.

Just as in the case of the one-dimensional $J_{1}-J_{2}$ model this model also has a simple exact dimer-singlet product ground state in a certain parameter region. However, by contrast with the one-dimensional $J_{1}-J_{2}$ model the dimer-singlet product ground state of the ShastrySutherland model is built up of a product of dimer singlets located on the next-nearest $J_{2}$ bonds and does not break the translational symmetry. 
Fig. 5 The nearest-neighbor bonds (solid lines) of strength $J_{1}$ and the next-nearest-neighbor diagonal bonds (dashed lines) of strength $J_{2}$ for the

Shastry-Sutherland model. The geometric unit cell is shown by the square with the grey dotted lines

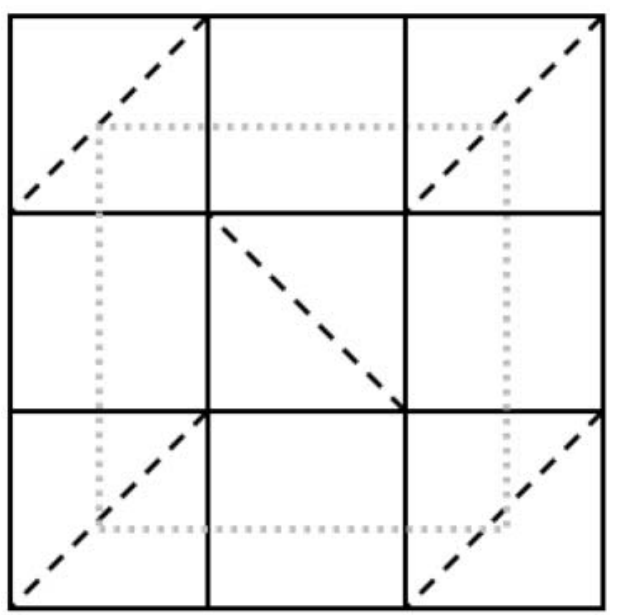

This model has been treated previously by Schwinger boson mean-field theory [13], exact diagonalization of small lattices [12, 14, 15], series expansions [16-19], the renormalization group [19], a gauge-theoretical approach [20], and the CCM [25, 86]. A recent review can be found in Ref. [21]. We know from these studies that the physics of the quantum model is similar to that of its classical counterpart for small $J_{2}<J_{1}$, i.e., we have semi-classical Néel long-range order. Furthermore, we know [11] that a simple dimersinglet product state given by dimer singlets on the diagonal bonds indicated by the dashed lines in Fig. 5 is the quantum ground state for large $J_{2}$. The energy per site of this dimersinglet product state is $E_{\text {dimer }} / N=-3 J_{2} / 8$. It becomes the ground state for $J_{2}>J_{2}^{c}$ where $J_{2}^{c} \approx(1.465 \pm 0.025) J_{1}$. Note that, by contrast to the one-dimensional $J_{1}-J_{2}$ model, the transition to the dimerized phase in the Shastry-Sutherland model is most likely of first order.

The application of the CCM to this model has been discussed at length in Ref. [25]. The interested reader is referred to this reference for more details about both the model and the details of the applying the CCM to it. We note that the CCM solution for the Néel model state with nearest-neighbor spin antiparallel was identified and this worked well in the region of $J_{2} / J_{1}<1.6$. This case has a Hamiltonian similar to that of (8), the nearest-neighbor bonds $J_{1}$ (solid lines) and next-nearest-neighbor bonds $J_{2}$ (dashed lines) run over those sites on the square lattice, as opposed to the linear chain for the case presented above.

However, we will show that a simple dimerized product ket state solution to the CCM equations also exists. Note firstly that we may define a collinear independent-spin product model state. This is a model state in which next-nearest-neighbor spins are antiparallel. This state is relevant for large antiferromagnetic $J_{2}$, since antiparallel next-nearest-neighbor spins satisfy the $J_{2}$ bonds. Thus, the "up" and "down" spins form alternate neighboring columns (or rows), see e.g. Refs. [88-92]. We choose the former case and call the corresponding model state the "columnar model state'. We rotate the "up" spins into (nominally) "down" as illustrated in Sect. 2.1, although we must now also take the columnar form of the model state into account. The relevant Hamiltonian in the appropriate local axes described above is given by 


$$
\begin{aligned}
H= & -J_{1} \sum_{\left\langle i_{x}, j_{x}\right\rangle}\left(s_{i_{x}}^{z} s_{j_{x}}^{z}+\frac{1}{2}\left\{s_{i_{x}}^{-} s_{j_{x}}^{-}+s_{i_{x}}^{+} s_{j_{x}}^{+}\right\}\right) \\
& +J_{1} \sum_{\left\langle i_{y}, j_{y}\right\rangle}\left(s_{i_{y}}^{z} s_{j_{y}}^{z}+\frac{1}{2}\left\{s_{i_{y}}^{-} s_{j_{y}}^{+}+s_{i_{y}}^{+} s_{j_{y}}^{-}\right\}\right) \\
& -J_{2} \sum_{\langle\langle i, k\rangle\rangle}\left(s_{i}^{z} s_{k}^{z}+\frac{1}{2}\left\{s_{i}^{-} s_{k}^{-}+s_{i}^{+} s_{k}^{+}\right\}\right),
\end{aligned}
$$

where the sum on $\left\langle i_{x}, j_{x}\right\rangle$ runs over all nearest-neighbor pairs of the lattice sites in the row or $x$-direction and the sum on $\left\langle i_{y}, j_{y}\right\rangle$ runs over all nearest-neighbor pairs of the lattice sites in the column or $y$-direction. Furthermore, the sum on $\langle\langle i, k\rangle\rangle$ runs over distinct next-nearestneighbor pairs of sites connected by the broken lines in Fig. 5 . We count each bond once and once only (for both the nearest-neighbor and next-nearest-neighbor bonds). The unit cell for this model contains four sites, and it is shown also in Fig. 5. Once again, in what follows we set $J_{1}=1$ and treat $J_{2}>0$ as the parameter of interest in the model.

At $J_{2}=0$, we have the usual square-lattice antiferromagnet, and the system is Néelordered at this point and in a finite range for $J_{2}>0$. Furthermore, we note that the ground state becomes the simple dimer-singlet product state for $J_{2}>J_{2}^{c}$ where $J_{2}^{c} \approx$ $(1.465 \pm 0.025) J_{1}$. We are able to define CCM correlation coefficients for spin dimers located on the diagonal bonds of the dashed lines of Fig. 5 with respect to the columnar model state. By setting these ket-state correlation coefficients to unity and all other CCM multispin correlation coefficients to zero, we are able to form the relevant dimer-singlet product state that is the exact ground state in this regime. We find that this CCM dimer solution is a stable solution for the CCM equations for all values of $J_{2} / J_{1}>0$, and this is because the dimersinglet product state is a true eigenstate for any values of $J_{1}$ and $J_{2}$. However, the energy of this state is low enough for it to become the ground-state energy only for large $J_{2}$. The CCM dimer solution yields also the correct exact energy for the dimer-singlet product state, namely, $E_{g} / N=-0.375 J_{2}$. The CCM ground-state energies are now shown for both the dimer and usual Néel results in Fig. 6. We see that there is a crossing of the Néel and dimer energies at $J_{2} / J_{1} \approx 1.48$, as reported in Ref. [79].

Another interesting point is that the bra-state correlation coefficients do not remain constant with respect to varying $J_{2}$. Indeed, we find many of the bra-state correlation coefficients diverge at a CCM critical point as may be seen in Fig. 7 for the next-nearest-neighbor brastate correlation coefficients on the diagonals (i.e., those corresponding to the dashed lines in Fig. 5). This is a critical point for the CCM bra-state equations only. The ket-state equations clearly do not contain a similar critical point. Hence, this is a critical point that is "driven" by the bra state alone. The critical points occur at values for $J_{2} / J_{1}$ of $1.059,1.243$, and 1.397 for the SUB2-4, LSUB4, and LSUB6 levels of approximation, respectively. This is in agreement with the position of the phase transition point at $J_{2}^{c} \approx(1.465 \pm 0.025) J_{1}$. We note that the CCM ket state is an exact ground eigenstate in this regime, whereas the bra state is not. As mentioned before, this is because the ket states and bra states are not explicitly constrained to be Hermitian conjugates of each other in the CCM parametrizations.

The results for the sublattice magnetization $M$ (with respect to the columnar model state) are shown in Fig. 8. We see that the values for the sublattice magnetization are negative for all values of $J_{2} / J_{1}$. Note that the true values for the sublattice magnetization are actually zero in this regime. Negative values of $M$ might be an indication of missing magnetic longrange order. However, we note that although the CCM results are negative, they remain close to zero (e.g., $|M|<10^{-2}$ at the LSUB6 level of approximation) for $J_{2} / J_{1}>2$. This indicates 


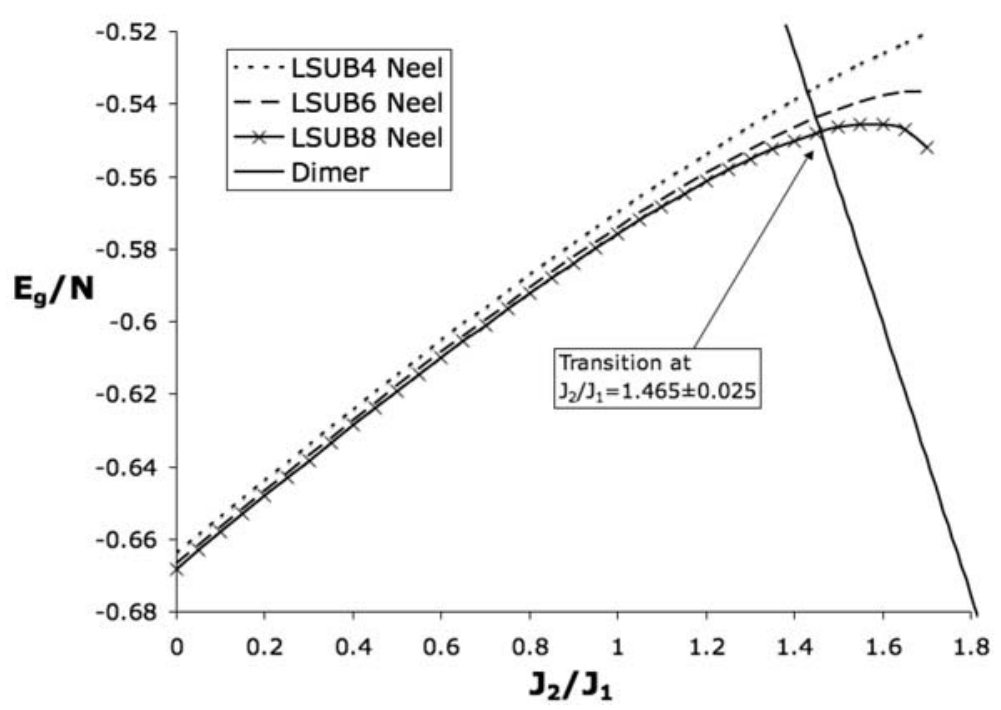

Fig. 6 The CCM ground-state energy for the spin-half Shastry-Sutherland model (with $J_{1}=1$ ) using the Néel model state (LSUB4, LSUB6, LSUB8) and the columnar model state. Note that in the latter case the exact ground-state energy is obtained for any LSUB $m$ with $m \geq 2$

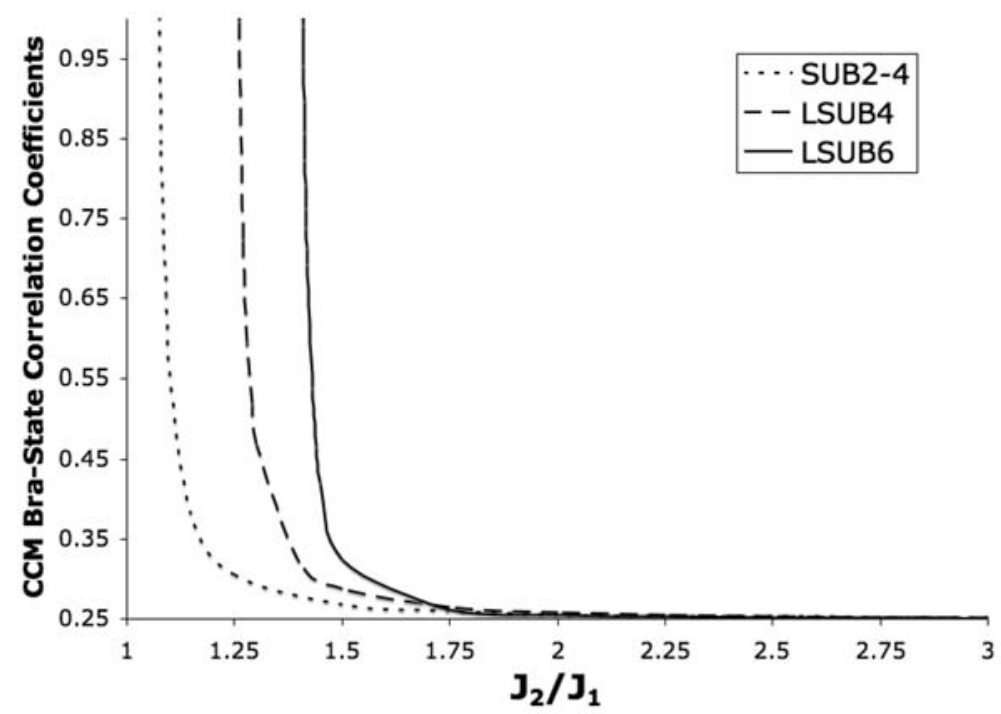

Fig. 7 CCM results using the columnar model state for the ground-state bra-state correlation coefficients for those sites connecting the dimers in the spin-half Shastry-Sutherland model

that we obtain generally good results for the sublattice magnetization. However, we see from Fig. 8 that our results clearly become worse for $J_{2} / J_{1}<2$. For example, we note that the sublattice magnetization diverges as we approach the CCM critical point (of the bra-state equations only) at $J_{2}^{c} / J_{1}$. We note that the bra and ket states are not explicitly constrained to be Hermitian conjugates. Hence, the bra state does not have to be an exact eigenstate 


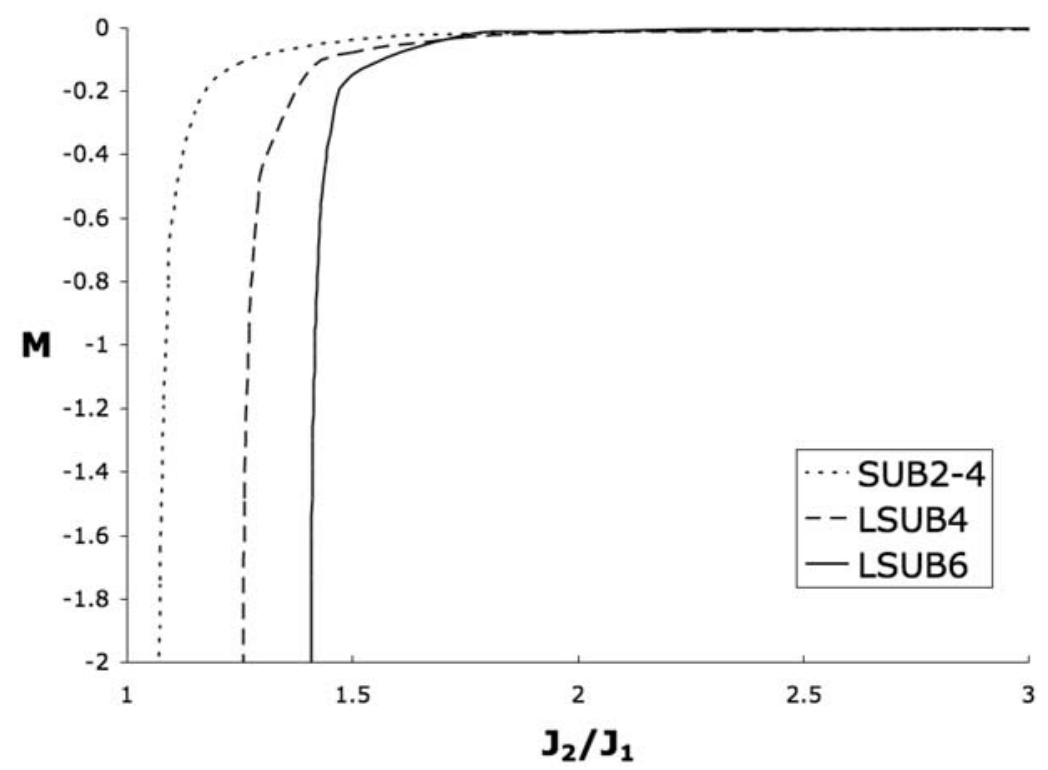

Fig. 8 The sublattice magnetization for the spin-half Shastry-Sutherland model for the columnar model state

of the Hamiltonian even though the ket state is for the Shastry-Sutherland model. We note therefore that this is enough to allow the bra-state equations to become critical even though the ket-state equations do not; hence, the nearest-neighbour bra-state correlation coefficient $\left(\tilde{\mathcal{S}}_{2}^{a}\right)$ diverges. The sublattice magnetization $\left(M=\frac{1}{2}-2 \tilde{\mathcal{S}}_{2}^{a}\right)$ therefore diverges also. Again, this is a reflection of the critical point that is "driven" by the bra state alone. The Néel state with nearest-neighbor spins antiparallel is the appropriate CCM model state [25] below the critical point $J_{2}<J_{2}^{c}$.

\subsection{The $J-J^{\prime}$ Heisenberg Antiferromagnet on the CAVO Lattice}

In this section, we consider an antiferromagnetic Heisenberg model in which the basic geometric unit cell contains four neighboring lattice sites on the underlying crystallographic lattice of the magnetic material $\mathrm{CaV}_{4} \mathrm{O}_{9}$ (CAVO), shown in Fig. 9. There are two nonequivalent antiferromagnetic nearest-neighbor bonds $J$ and $J^{\prime}$ belonging to dimers $\left(J^{\prime}\right)$ and to four-spin plaquettes $(J)$ respectively. The ground state of the quantum model depends on the ratio $J^{\prime} / J$ of the competing bonds. Using a unit cell as defined in Fig. 9, the plaquette bonds $J$ are inside the four-site unit cell and the dimer bonds $J^{\prime}$ connect sites in different unit cells. We note that this model is not frustrated but the two non-equivalent nearest-neighbor bonds lead to a competition in the quantum system. Henceforth, we choose an energy scale such that $J=1$.

We note that several techniques [28-34] suggest that the Néel-ordered ground state at $J^{\prime} / J=1$ persists over a finite range of values of $J^{\prime} / J$ around this point, $J_{c_{1}}^{\prime} / J<J^{\prime} / J<$ $J_{c_{2}}^{\prime} / J$. The best estimates for $J_{c_{1}}^{\prime} / J$ and $J_{c_{2}}^{\prime} / J$ are probably provided by quantum Monte Carlo calculations $[29,30]$ that suggest that the range over which Néel order is stable is given by $J_{c_{1}}^{\prime} / J \approx 0.939$ and $J_{c_{2}}^{\prime} / J \approx 1.68 \pm 0.14$. For $J^{\prime} / J<J_{c_{1}}^{\prime} / J$ the model exhibits a quadrumerized plaquette VBC phase with enhanced spin correlations on the four-spin 
Fig. 9 The CAVO lattice. The nearest-neighbor bonds that connect two sites on a four-site plaquette are shown by the solid lines and have a bond strength given by $J(=1)$. The nearest-neighbor bonds that connect two sites on different plaquettes (dimer bonds) are shown by the dotted lines and have a bond strength given by $J^{\prime}$. The unit cell of the lattice is shown by the square with the grey dashed lines

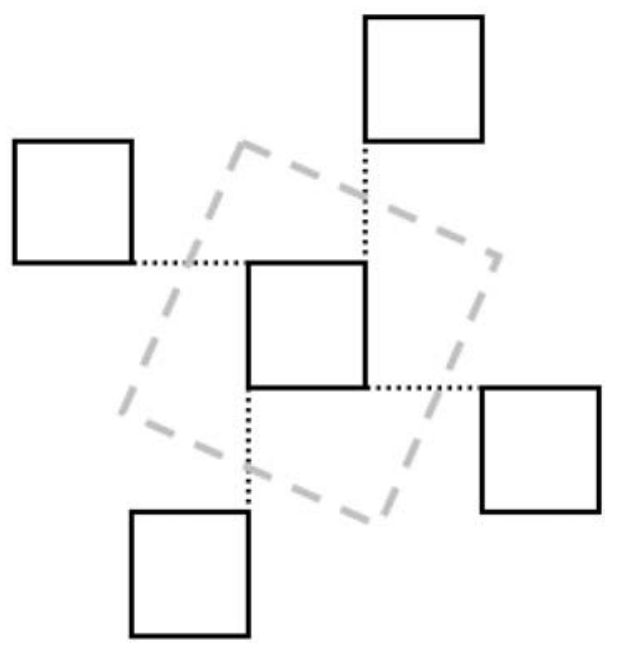

plaquettes, and for $J^{\prime} / J>J_{c_{2}}^{\prime} / J$ it has a dimerized VBC phase with enhanced spin correlations on the dimers. Neither of the VBC phases breaks the translational symmetry of the lattice. Furthermore, we mention that by contrast with the previously considered models (see Sects. 3.1 and 3.2) this model has no simple exact product ground state for any value of $J^{\prime} / J$. The interested reader can find more information on the ground-state phases in Refs. [28-34].

The four-site plaquettes in the unit cell become decoupled only in the limit $J^{\prime} / J=0$. The ground state is a product of such four-site plaquette singlets in this limit. To model such a state using the CCM we start again from the Néel model state; namely, a state in which the spins on nearest-neighbor sites are antiparallel.

To create an exact plaquette-singlet product VBC ground state at $J^{\prime} / J=0$ using the $\mathrm{CCM}$ we have to adjust the nearest-neighbor correlation coefficients $\mathcal{S}_{2}^{a}$ and $\mathcal{S}_{2}^{b}$ and a single four-body plaquette correlation coefficient $\mathcal{S}_{4}^{p}$ containing all four sites properly. (Note that $\mathcal{S}_{2}^{a}$ represents those ket-state coefficients for the nearest-neighbor two-body cluster connecting sites on a plaquette indicated by the solid lines in Fig. 9, whereas $\mathcal{S}_{2}^{b}$ represents those ket-state coefficients for the nearest-neighbor two-body cluster connecting sites on a dimer indicated by the dotted lines in the same figure. The coefficient $\mathcal{S}_{4}^{p}$ represents those ket-state coefficients for the four-body cluster corresponding to a plaquette indicated by the solid lines in Fig. 9.) Indeed, it is easy to show that setting the ket-state correlation coefficients $\mathcal{S}_{2}^{a}$ and $\mathcal{S}_{4}^{p}$ to a value of 0.5 and all other ket-state correlation coefficients (including $\mathcal{S}_{2}^{b}$ ) to zero the plaquette-singlet product VBC state is obtained exactly, see Fig. 10. We are able to track this plaquette solution as $J^{\prime} / J$ is increased away from the point $J^{\prime} / J=0$ where it is exact. Furthermore, we are also able to reproduce exactly the dimer-singlet product ground state in the limit $J^{\prime} / J \rightarrow \infty$. In this limit, the nearest-neighbor ket-state correlation coefficient $\mathcal{S}_{2}^{b}$ on the dimer bonds (dotted lines in Fig. 9) has a value of one and all other coefficients (e.g., $\mathcal{S}_{2}^{a}$ and $\mathcal{S}_{4}^{p}$ ) are zero, see Fig. 10 . This solution is produced automatically when we track the solution (outlined above) from $J^{\prime} / J=0$, and so our CCM Ansatz produces accurate results in all phases of this model. The corresponding bra-state correlation coefficients $\tilde{\mathcal{S}}_{2}^{a}, \tilde{\mathcal{S}}_{2}^{b}$ and $\tilde{\mathcal{S}}_{4}^{p}$ behave smoothly in the entire range of $J^{\prime} / J$, see Fig. 11 .

Results for the ground-state energy are shown in Fig. 12. We see that the exact groundstate energy at $J^{\prime} / J=0$ is reproduced for LSUB $m$ levels of approximation with $m \geq 4$, as expected. At $J^{\prime} / J=1$, we reproduce a previous result [82] using the Néel model state for 


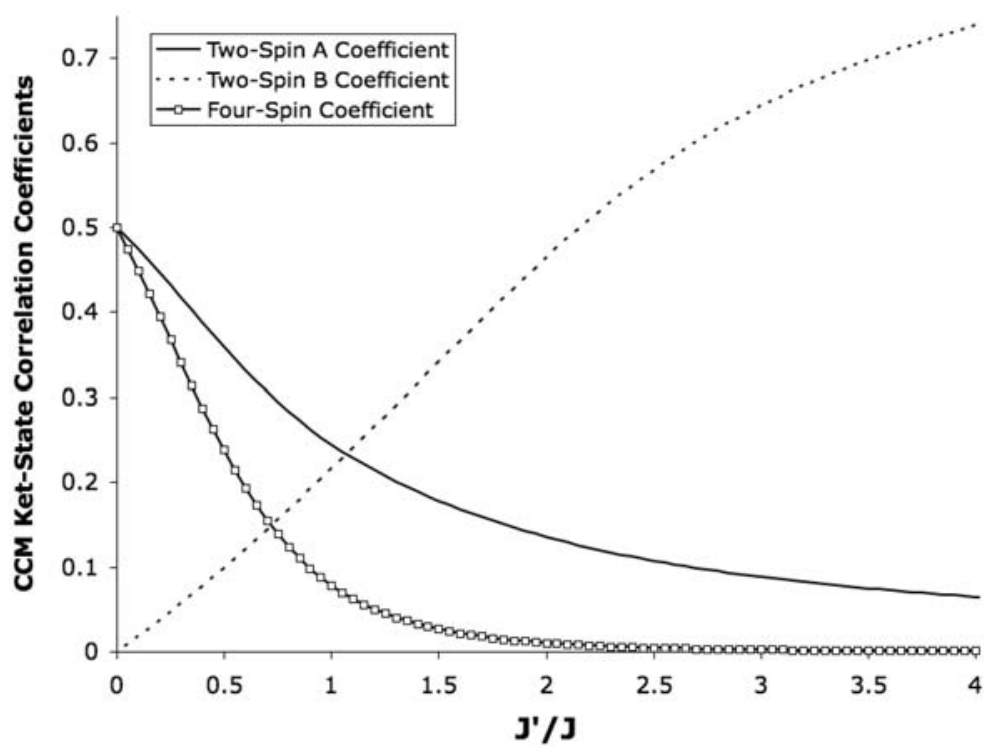

Fig. 10 CCM results at the LSUB8 level of approximation for the ground-state nearest-neighbor two-spin $A$ and $B$ ket-state coefficients $\left(\mathcal{S}_{2}^{a}\right.$ and $\left.\mathcal{S}_{2}^{b}\right)$ and the plaquette four-spin ket-state correlation $\left(\mathcal{S}_{4}^{p}\right)$ coefficients of the spin-half antiferromagnet on the CAVO lattice

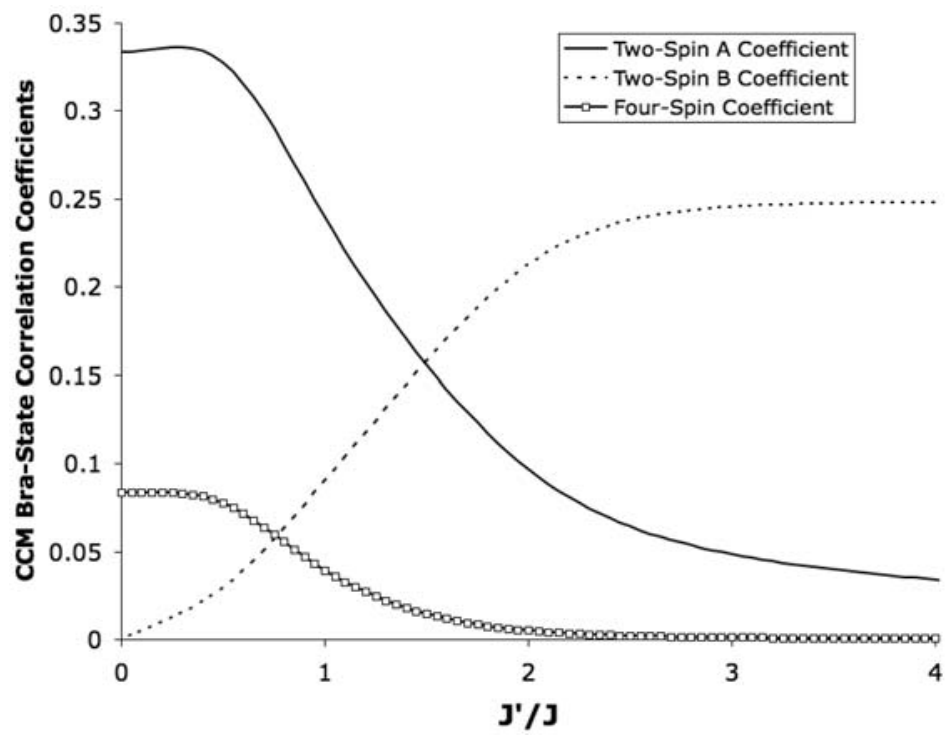

Fig. 11 CCM results at the LSUB8 level of approximation for the ground-state nearest-neighbor two-spin $A$ and $B$ bra-state coefficients $\left(\tilde{\mathcal{S}}_{2}^{a}\right.$ and $\left.\tilde{\mathcal{S}}_{2}^{b}\right)$ and the plaquette four-spin bra-state $\left(\tilde{\mathcal{S}}_{4}^{p}\right)$ bra-state correlation coefficients of the spin-half antiferromagnet on the CAVO lattice

this CAVO lattice. At all values of $J^{\prime} / J$, the LSUB $m$ results are seen to converge rapidly with increasing levels of $\mathrm{LSUB} m$ approximation. We note that the system should again 


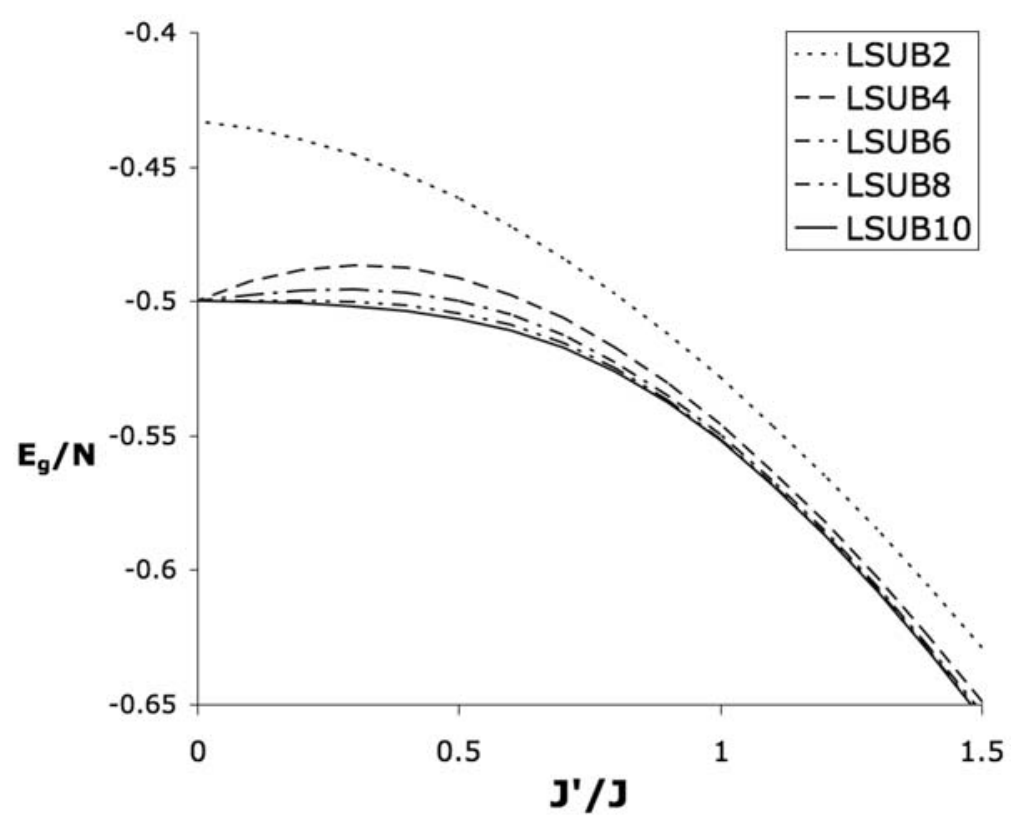

Fig. $12 \mathrm{CCM}$ results for the ground-state energy of the $J-J^{\prime}$ Heisenberg antiferromagnet on the CAVO lattice (with $J=1$ )

decouple into dimers as $J^{\prime} / J \rightarrow \infty$ and the correct ground-state energy $\left(-0.375 J^{\prime}\right)$ is reproduced in this limit. The CCM provides excellent results for the ground-state energy for all values of $J^{\prime} / J$.

The results for the sublattice magnetization are shown in Fig. 13. We extrapolate the raw LSUB $m$ data to the limit $m \rightarrow \infty$ in order to determine the quantum phase transition points where the magnetic Néel long-range order vanishes. An appropriate extrapolation rule for the magnetic order parameter for systems showing a ground-state order-disorder transition is [87-92] $M(n)=b_{0}+b_{1}(1 / n)^{1 / 2}+b_{2}(1 / n)^{3 / 2}$, where we use LSUB $m$ results with $m=\{4,6,8,10\}$. The results for these quantum critical points are shown in Table 2 . Again, we note that these results indicate that the Néel order persists over a finite range around $J^{\prime} / J=1$ for the of the $J-J^{\prime}$ Heisenberg antiferromagnet on the CAVO lattice. Finally, these results show that the CCM can handle plaquette VBC ordering as easily as dimer VBC ordering or the usual Néel ordering. Furthermore, we have demonstrated that quantum critical points can be determined by using the CCM to high orders of LSUBm approximation.

\section{Conclusions}

We have shown in this article that we can easily form dimer and plaquette VBC ground states using the CCM with independent-spin product model states. We have investigated a number of cases in which the ground state was a product of localized dimer or plaquette singlets. Firstly, we considered the spin-half $J_{1}-J_{2}$ model for the linear chain. We showed that we are able to reproduce exactly the dimerized ground state at $J_{2} / J_{1}=0.5$. Interestingly, a symmetry-breaking dimerized CCM solution is observed for $J_{2} / J_{1}<0.5$, which 


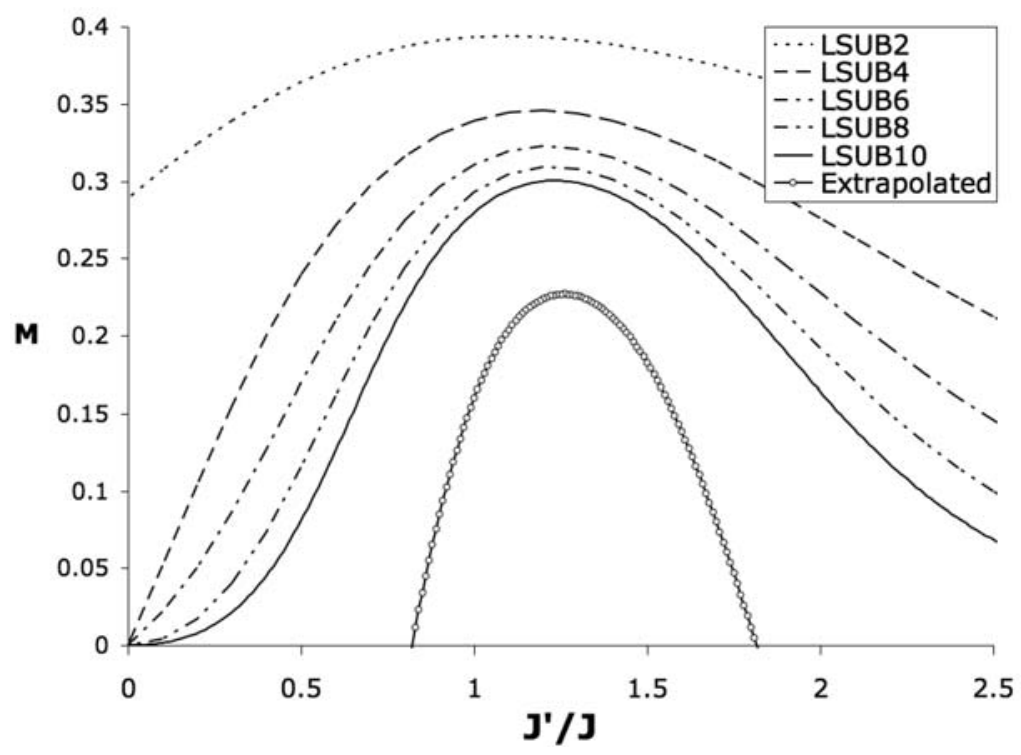

Fig. $13 \mathrm{CCM}$ results for the ground-state sublattice magnetization $M$ of the $J-J^{\prime}$ Heisenberg antiferromagnet on the CAVO lattice

Table 2 Results for the quantum critical points of the $J-J^{\prime}$ Heisenberg antiferromagnet on the CAVO lattice

\begin{tabular}{lll}
\hline Method & $J_{c_{1}}^{\prime} / J$ & $J_{c_{2}}^{\prime} / J$ \\
\hline CCM Extrapolation $(m=\{4,6,8,10\})$ & 0.82 & 1.82 \\
QMC [29, 30] & 0.939 & $1.68 \pm 0.14$ \\
Cumulant Series Expansions [32] & 0.9 & 1.7 \\
Non-Linear Spin-Wave Theory [34] & 0.90 & 1.6 \\
Schwinger-Boson Mean-Field Theory [33] & 0.6 & 2.4 \\
\hline
\end{tabular}

only becomes equal to the usual ('Néel-type') solution that conserves the lattice symmetry at a CCM critical point $J_{2} /\left.J_{1}\right|_{c_{1}}$. Results for the bra state correlation coefficients diverged at this point also. We took this to indicate the onset of the dimerized ground-state phase that breaks the translational lattice symmetry. We found that the dimerized phase extends over a finite range of values of $J_{2} / J_{1}$ both above and below 0.5 . Results for the ground-state energy for the dimerized CCM solution were found to agree extremely well with the results of exact diagonalizations for $N=28$ and $N=32$ chains in the dimerized regime. The change from the usual ('Néel-type') solution to the dimerized solution was also observed in the behavior of the sublattice magnetization.

We then considered the Shastry-Sutherland model and we demonstrated that the CCM can span the correct ground states in both the Néel and the dimerized phases. We found a CCM critical point for the dimerized solution that is "driven" by the CCM bra state alone. Furthermore, the position of this critical point is in good agreement with the known value for the phase transition point of this model. Results for the sublattice magnetization (that ought to be zero in the dimerized phase) were found to be generally good in the dimerized regime. For example, results for the sublattice magnetization at the LSUB6 level of approximation 
were found to be $|M|<10^{-2}$ for $J_{2} / J_{1}>2$. However, the sublattice magnetization was also found to be negative and to diverge at the critical point of the bra-state equations.

Finally, we considered a spin-half Heisenberg antiferromagnet with nearest-neighbor bonds with respect to an underlying lattice that corresponds to that of the magnetic material $\mathrm{CaV}_{4} \mathrm{O}_{9}$. The four nearest-neighbor bonds that connect sites on a four-site plaquette have a bond strength given by $J$ and the nearest-neighbor bonds that connect sites belonging to different plaquettes (dimer bonds) have a bond strength given by $J^{\prime}$. The exact plaquettesinglet ground state at $J^{\prime} / J=0$ and the exact dimer-singlet ground state as $J^{\prime} / J \rightarrow \infty$ were both reproduced exactly using the CCM with the same choice of Néel model state. We found that the CCM can provide precise results for the ground-state energy over all intervening values of the parameter $J^{\prime} / J$. Results for the sublattice magnetization were presented, and these results indicated that the Néel-ordered regime persists over a finite range of values of $J^{\prime} / J$ around the point $J^{\prime} / J=1$. For large (and small) values of $J^{\prime} / J$, the Néel long-range order is destroyed by local singlet formation on dimers (and on plaquettes). Extrapolations of LSUB $m$ data suggest that the Néel-order regime extends over the range $0.82<J^{\prime} / J<1.82$. These results were found to be in fairly reasonable agreement with quantum Monte Carlo results for this model [29, 30]. However, a discussion of the accuracy of phase transition points estimated using the CCM is beyond the scope of this article and so will form the contents of another article, although we note here that we believe also that higher orders of LSUB $m$ approximation would provide closer agreement.

As noted above, the CCM is one of the most powerful and most widely applied techniques of quantum many-body theory. One of the reasons for this success is based on the fact that the CCM allows the inclusion of multi-particle correlations into the ground- and excited-state wave functions in a controlled and systematic manner. The range of applicability of the CCM to lattice quantum spin systems has been greatly extended previously by the creation of efficient and powerful high-order computer codes for independent-spin product (e.g. Néel) model states. These codes are simple to use and they are generally accurate in practical applications. Furthermore, they are extremely flexible in terms of defining and solving new spin problems.

Previously however, non-classical orderings (such as local singlet formation) have also been considered using the CCM by employing non-Néel model states. This typically involved the direct use of products of, e.g., local dimer singlets, in the model state. However, this approach required a whole new matrix-operator formalism to be created for each new problem [62]. This is usually tedious and time-consuming, although it is normally straightforward mathematically. More importantly, however, the existing high-order CCM codes would then need to be amended extensively also in order to implement the new matrix algebra for each new problem. Here we have presented a much simpler and more universal approach that combines exact solutions for dimer or plaquette VBC product ground states with the computational implementation described in Refs. [64, 68, 72, 77, 80, 81] based on independent-spin product model states.

One seeming shortcoming of this new approach was found to be that the ket state can be an exact representation of the true ground state, whereas the bra state might not be at the same level of LSUBm approximation. This is due to the simple fact that the NCCM parametrizations of the ket and bra wave functions are not manifestly Hermitian conjugates of each other. This meant, for example, that the exact ground-state energy of the Shastry-Sutherland model in the dimerized phase was reproduced, since it is calculated from the ket-state correlation coefficients alone, whereas the exact sublattice magnetization (known to be zero in this regime) was not, since its calculation requires the use of the bra-state correlation coefficients as well as the ket-state coefficients. We speculate that this problem might be overcome 
by employing the extended coupled cluster method (ECCM) that contains an exponentiated form of the bra-state correlation operator in the (ground) bra state. We believe that it is possible to construct a similar exact bra-state solution for the ECCM as we have constructed here for the ket state using the NCCM based on independent-spin product model states.

As noted above, an alternative approach is to utilize model states that are formed directly from products of local dimer or plaquette states (even for the NCCM). In this case, the ket and bra state can become Hermitian conjugates (trivially) when the model state is the exact ground state. Indeed, in this case, all of the CCM ket- and bra-state correlation coefficients become zero. However, even here, we note that the lack of manifest Hermiticity is a general feature of the CCM, i.e., one that can exist even for results generated by calculations based on such valence-bond model states. Indeed, in those cases for which the model state is not (trivially) the ground state, the bra and ket states are again not constrained manifestly to be Hermitian conjugates at any given level of LSUB $m$ approximation.

In conclusion, this new approach for dimer- and plaquette-ordered ground states is flexible, simple to implement, and very powerful. Lattices of arbitrary complexity can also be treated using this new method. Furthermore, this approach is simple because we are using generally independent-spin product model states derived from classical ground states. Indeed, this is far simpler than the alternative of creating a whole new matrix formalism for each new model state formed from products of localized states. Finally, this approach is powerful because the high-order codes based on independent-spin product model states, which have been employed previously with great success, may be used directly in order to simulate the properties of these non-Néel states. The useful LSUB $m$ and SUB $n$ approximation schemes devised for the Néel model states may be used directly also. The results of the LSUB $m$ scheme may be extrapolated easily to the limit $m \rightarrow \infty$ using existing 'heuristic' extrapolation schemes. The results presented here offer a great enhancement to the range of applicability of the CCM for lattice quantum spin systems that demonstrate 'novel states' of quantum order.

Acknowledgements One of us (DJJF) gratefully acknowledges support for the research presented here from the European Science Foundation (Research Network Programme: Highly Frustrated Magnetism). Two of us (RZ and JR) thank the DFG for support (project RI615/16-1).

\section{References}

1. Majumdar, C.K., Ghosh, D.K.: J. Math. Phys. 10, 1388 (1969)

2. Majumdar, C.K., Ghosh, D.K.: J. Math. Phys. 10, 1399 (1969)

3. Tonegawa, T., Harada, I.: J. Phys. Soc. Jpn. 56, 2153 (1987)

4. Nomura, K., Okamoto, K.: Phys. Lett. 169A, 433 (1992)

5. Nomura, K., Okamoto, K.: J. Phys. Soc. Jpn. 62, 1123 (1993)

6. Nomura, K., Okamoto, K.: J. Phys. A: Math. Gen. 27, 5773 (1994)

7. Chitra, R., Pati, S., Krishnamurthy, H.R., Sen, D., Ramasesha, S.: Phys. Rev. B 52, 6581 (1995)

8. White, S.R., Affleck, I.: Phys. Rev. B 54, 9862 (1996)

9. Mikeska, H.-J., Kolezhuk, A.K.: In: Schollwöck, U., Richter, J., Farnell, D.J.J., Bishop, R.F. (eds.) Quantum Magnetism. Lecture Notes in Physics, vol. 645, pp. 1-83. Springer, Berlin (2004)

10. Aligia, A.A., Batista, C.D., Eßler, F.H.L.: Phys. Rev. B 62, 3259 (2000)

11. Shastry, B.S., Sutherland, B.: Physica B 108, 1069 (1981)

12. Richter, J., Schulenburg, J., Honecker, A.: In: Schollwöck, U., Richter, J., Farnell, D.J.J., Bishop, R.F. (eds.) Quantum Magnetism. Lecture Notes in Physics, vol. 645, pp. 85-183. Springer, Berlin (2004)

13. Albrecht, M., Mila, F.: Europhys. Lett. 34, 145 (1996)

14. Zheng, W., Oitmaa, J., Hamer, C.J.: Phys. Rev. B 65, 014408 (2002)

15. Läuchli, A., Wessel, S., Sigrist, M.: Phys. Rev. B 66, 014401 (2002)

16. Zheng, W., Hamer, C.J., Oitmaa, J.: Phys. Rev. B 60, 6608 (1999) 
17. Müller-Hartmann, E., Singh, R.R.P., Knetter, C., Uhrig, G.S.: Phys. Rev. Lett. 84, 1808 (2000)

18. Koga, A., Kawakami, N.: Phys. Rev. Lett. 84, 4461 (2000)

19. Al Hajii, M., Guihery, N., Malrieu, J.-P., Bouquillon, B.: Eur. Phys. J. B 41, 11 (2004)

20. Chung, C.H., Marston, J.B., Sachdev, S.: Phys. Rev. B 64, 134407 (2001)

21. Miyahara, S., Ueda, K.: J. Phys.: Condens. Matter 15, R327 (2003)

22. Miyahara, S., Ueda, K.: Phys. Rev. Lett. 82, 3701 (1999)

23. Albrecht, M., Mila, F.: Europhys. Lett. 34, 145 (1996)

24. Kageyama, H., Yoshimura, K., Stern, R., Mushnikov, N.V., Onizuka, K., Kato, M., Kosuge, K., Slichter, C.P., Goto, T., Ueda, Y.: Phys. Rev. Lett. 82, 3168 (1999)

25. Darradi, R., Richter, J., Farnell, D.J.J.: Phys. Rev. B 72, 104425 (2005)

26. Richter, J., Schulenburg, J., Honecker, A., Schmalfuß, D.: Phys. Rev. B 70, 174454 (2004)

27. Misguich, G., Sindzingre, P.: J. Phys.: Condens. Matter 19, 145202 (2007)

28. Ueda, K., Kontani, H., Sigrist, M., Lee, P.A.: Phys. Rev. Lett. 76, 1932 (1996)

29. Troyer, M., Kontani, H., Ueda, K.: Phys. Rev. Lett. 76, 3822 (1996)

30. Troyer, M., Imada, M., Ueda, K.: J. Phys. Soc. Jpn. 66, 2957 (1997)

31. Gelfand, M.P., Zheng, W., Singh, R.R.P., Oitmaa, J., Hamer, C.J.: Phys. Rev. Lett. 77, 2794 (1996)

32. Zheng, W., Gelfand, M.P., Singh, R.R.P., Oitmaa, J., Hamer, C.J.: Phys. Rev. B 55, 11377 (1997)

33. Albrecht, M., Mila, F.: Phys. Rev. B 53, R2945 (1996)

34. Meshkov, S.V., Foerster, D.: J. Phys.: Condens. Matter 8, 7917 (1996)

35. Singh, R.R.P., Gelfand, M.P., Huse, D.A.: Phys. Rev. Lett. 61, 2484 (1988)

36. Ivanov, N.B., Krüger, S.E., Richter, J.: Phys. Rev. B 53, 2633 (1996)

37. Krüger, S.E., Richter, J., Schulenberg, J., Farnell, D.J.J., Bishop, R.F.: Phys. Rev. B 61, 14607 (2000)

38. Tomczak, P., Richter, J.: J. Phys. A: Math. Gen. 34, L461 (2001)

39. Darradi, R., Richter, J., Farnell, D.J.J.: J. Phys.: Condens. Matter 17, 341 (2005)

40. Wenzel, S., Bogacz, L., Janke, W.: Phys. Rev. Lett. 101, 127202 (2008)

41. Coester, F.: Nucl. Phys. 7, 421 (1958)

42. Coester, F., Kümmel, H.: Nucl. Phys. 17, 477 (1960)

43. Čižek, J.: J. Chem. Phys. 45, 4256 (1966)

44. Čižek, J.: Adv. Chem. Phys. 14, 35 (1969)

45. Bishop, R.F., Lührmann, K.H.: Phys. Rev. B 17, 3757 (1978)

46. Bishop, R.F., Lührmann, K.H.: Phys. Rev. B 26, 5523 (1982)

47. Kümmel, H., Lührmann, K.H., Zabolitzky, J.G.: Phys. Rep. 36C, 1 (1978)

48. Arponen, J.S.: Ann. Phys. (NY) 151, 311 (1983)

49. Bishop, R.F., Kümmel, H.: Phys. Today 40(3), 52 (1987)

50. Arponen, J.S., Bishop, R.F., Pajanne, E.: Phys. Rev. A 36, 2519 (1987)

51. Arponen, J.S., Bishop, R.F., Pajanne, E.: Phys. Rev. A 36, 2539 (1987)

52. Arponen, J.S., Bishop, R.F., Pajanne, E.: In: Vashishta, P., Kalia, R.K., Bishop, R.F. (eds.) Condensed Matter Theories, vol. 2, p. 357. Plenum, New York (1987)

53. Bartlett, R.J.: J. Phys. Chem. 93, 1697 (1989)

54. Bishop, R.F.: Theor. Chim. Acta 80, 95 (1991)

55. Roger, M., Hetherington, J.H.: Phys. Rev. B 41, 200 (1990)

56. Roger, M., Hetherington, J.H.: Europhys. Lett. 11, 255 (1990)

57. Bishop, R.F., Parkinson, J.B., Xian, Y.: Phys. Rev. B 44, 9425 (1991)

58. Bishop, R.F., Parkinson, J.B., Xian, Y.: Phys. Rev. B 46, 880 (1992)

59. Bishop, R.F., Parkinson, J.B., Xian, Y.: J. Phys.: Condens. Matter 5, 9169 (1993)

60. Farnell, D.J.J., Parkinson, J.B.: J. Phys.: Condens. Matter 6, 5521 (1994)

61. Bishop, R.F., Hale, R.G., Xian, Y.: Phys. Rev. Lett. 73, 3157 (1994)

62. Xian, Y.: J. Phys.: Condens. Matter 6, 5965 (1994)

63. Bursill, R., Gehring, G.A., Farnell, D.J.J., Parkinson, J.B., Xiang, T., Zeng, C.: J. Phys.: Condens. Matter 7, 8605 (1995)

64. Hale, R.G.: Ph.D. Thesis. UMIST, Manchester, UK (1995)

65. Bishop, R.F., Farnell, D.J.J., Parkinson, J.B.: J. Phys.: Condens. Matter 8, 11153 (1996)

66. Farnell, D.J.J., Krüger, S.A., Parkinson, J.B.: J. Phys.: Condens. Matter 9, 7601 (1997)

67. Bishop, R.F., Xian, Y., Zeng, C.: In: Ludeña, E.V., Vashishta, P., Bishop, R.F. (eds.) Condensed Matter Theories, vol. 11, p. 91. Nova Science, Commack (1996)

68. Zeng, C., Farnell, D.J.J., Bishop, R.F.: J. Stat. Phys. 90, 327 (1998)

69. Bishop, R.F., Farnell, D.J.J., Parkinson, J.B.: Phys. Rev. B 58, 6394 (1998)

70. Bishop, R.F.: In: Navarro, J., Polls, A. (eds.) Microscopic Many-Body Theories and Their Applications. Lecture Notes in Physics, vol. 510, p. 1. Springer, Berlin (1998)

71. Rosenfeld, J., Ligterink, N.E., Bishop, R.F.: Phys. Rev. B 60, 4030 (1999) 
72. Bishop, R.F., Farnell, D.J.J., Krüger, S.E., Parkinson, J.B., Richter, J., Zeng, C.: J. Phys.: Condens. Matter 12, 7601 (2000)

73. Bishop, R.F., Farnell, D.J.J., Ristig, M.L.: Int. J. Mod. Phys. B 14, 1517 (2000)

74. Farnell, D.J.J., Bishop, R.F., Gernoth, K.A.: Phys. Rev. B 63, 220402R (2001)

75. Farnell, D.J.J., Gernoth, K.A., Bishop, R.F.: Phys. Rev. B 64, 172409 (2001)

76. Krüger, S.E., Richter, J.: Phys. Rev. B 64, 024433 (2001)

77. Farnell, D.J.J., Bishop, R.F., Gernoth, K.A.: J. Stat. Phys. 108, 401 (2002)

78. Ivanov, N.B., Richter, J., Farnell, D.J.J.: Phys. Rev. B 66, 014421 (2002)

79. Farnell, D.J.J., Bishop, R.F.: arXiv:cond-mat/0311126

80. Krüger, S.E., Farnell, D.J.J., Richter, J.: Int. J. Mod. Phys. B 17, 5347 (2003)

81. For the numerical calculation we use the program package 'Crystallographic Coupled Cluster Method' of D.J.J. Farnell and J. Schulenburg, see http://www-e.uni-magdeburg.de/jschulen/ccm/index.html

82. Farnell, D.J.J., Schulenberg, J., Richter, J., Gernoth, K.A.: Phys. Rev. B 72, 172408 (2005)

83. Krüger, S.E., Darradi, R., Richter, J., Farnell, D.J.J.: Phys. Rev. B 73, 094404 (2006)

84. Schmalfuß, D., Darradi, R., Richter, J., Schulenburg, J., Ihle, D.: Phys. Rev. Lett. 97, 157201 (2006)

85. Farnell, D.J.J., Bishop, R.F.: arXiv:cond-mat/0606060

86. Richter, J., Darradi, R., Zinke, R., Bishop, R.F.: Int. J. Mod. Phys. B 21, 2273 (2007)

87. Zinke, R., Schulenburg, J., Richter, J.: Eur. Phys. J. B 61, 147 (2008)

88. Bishop, R.F., Li, P.H.Y., Darradi, R., Richter, J.: J. Phys.: Condens. Matter 20, 255251 (2008)

89. Bishop, R.F., Li, P.H.Y., Darradi, R., Schulenburg, J., Richter, J.: Phys. Rev. B 78, 054412 (2008)

90. Bishop, R.F., Li, P.H.Y., Darradi, R., Richter, J.: Europhys. Lett. 83, 47004 (2008)

91. Bishop, R.F., Li, P.H.Y., Darradi, R., Richter, J., Campbell, C.E.: J. Phys.: Condens. Matter 20, 415213 (2008)

92. Darradi, R., Derzhko, O., Zinke, R., Schulenburg, J., Krüger, S.E., Richter, J.: Phys. Rev. B 78, 214415 (2008) 